\title{
A Novel Angiotensin-(1-7) Glycosylated Mas Receptor Agonist for Treating Vascular Cognitive Impairment and Inflammation- Related Memory Dysfunction
}

\author{
Meredith Hay, (1) Robin Polt, Michael L. Heien, Todd W. Vanderah, Tally M. Largent-Milnes, \\ Kathleen Rodgers, Torsten Falk, Mitchell J. Bartlett, Kristian P. Doyle, and John P. Konhilas \\ Departments of Physiology (M.H., J.P.K.), Chemistry and Biochemistry (R.P., M.L.H.), Pharmacology (T.W.V., T.M.L.-M., K.R., \\ T.F., M.J.B.), Neurology (T.F., M.J.B.), and Immunobiology (K.P.D.), Evelyn F. McKnight Brain Institute (M.H.), Sarver Heart \\ Center (M.H., J.P.K.), and Center for Innovation in Brain Science (M.H., T.W.V., K.R.), University of Arizona, Tucson, Arizona
}

Received November 7, 2018; accepted January 29, 2019

\begin{abstract}
Increasing evidence indicates that decreased brain blood flow, increased reactive oxygen species (ROS) production, and proinflammatory mechanisms accelerate neurodegenerative disease progression such as that seen in vascular contributions to cognitive impairment and dementia (VCID) and Alzheimer's disease and related dementias. There is a critical clinical need for safe and effective therapies for the treatment and prevention of cognitive impairment known to occur in patients with VCID and chronic inflammatory diseases such as heart failure (HF), hypertension, and diabetes. This study used our mouse model of VCID/HF to test our novel glycosylated angiotensin-(1-7) peptide Ang-1-6-O-Ser-GlcNH2 (PNA5) as a therapy to treat VCID and to investigate circulating inflammatory biomarkers that may be involved. We demonstrate that PNA5 has greater brain penetration
\end{abstract}

compared with the native angiotensin-(1-7) peptide. Moreover, after treatment with $1.0 / \mathrm{mg} / \mathrm{kg}$, s.c., for 21 days, PNA5 exhibits up to 10 days of sustained cognitive protective effects in our VCID/HF mice that last beyond the peptide half-life. PNA5 reversed object recognition impairment in VCID/HF mice and rescued spatial memory impairment. PNA5 activation of the Mas receptor results in a dose-dependent inhibition of ROS in human endothelial cells. Last, PNA5 treatment decreased VCID/HF-induced activation of brain microglia/macrophages and inhibited circulating tumor necrosis factor $\alpha$, interleukin (IL)-7, and granulocyte cell-stimulating factor serum levels while increasing that of the anti-inflammatory cytokine IL-10. These results suggest that PNA5 is an excellent candidate and "first-inclass" therapy for treating VCID and other inflammation-related brain diseases.

\section{Introduction}

Increasing evidence suggest that decreased brain blood flow, increased reactive oxygen species (ROS) production and proinflammatory mechanisms accelerate the progression of neurodegenerative diseases such as vascular contributions to cognitive impairment and dementia (VCID) and patients with systemic inflammatory disease that are at risk for Alzheimer's disease (AD) and related dementias (Pullicino

This work was supported by the National Heart, Lung, and Blood Institute [Grants R01-HL-098256 and KO2-HL-10579] and National Institute of Neurological Disorders and Stroke [Grant R01-NS-091238]. M.H. is the founder and a major stockholder of and a scientific consultant for ProNeurogen, Inc., which holds exclusive licensing rights from the University of Arizona for the technology discussed herein. No other potential conflicts of interest relevant to this article are reported.

https://doi.org/10.1124/jpet.118.254854. et al., 2008; Santos et al., 2013, 2017; Corriveau et al., 2016; Gorelick et al., 2017; Pavol et al., 2018; Price et al., 2018). In older adults, heart failure (HF) results in decreased brain perfusion, and increased brain and systemic inflammation is known to increase the risk for the development of cognitive impairment and AD (Qiu et al., 2006; Cermakova et al., 2015; Love and Miners, 2016; Miners et al., 2016; Suzuki et al., 2016). There is a critical need for safe and effective therapies for the treatment and prevention of cognitive impairment known to occur in patients with VCID and chronic inflammatory diseases such as HF, hypertension, and diabetes. The ideal therapeutic candidate to treat inflammation-related neurodegenerative disease would be designed with criteria to interrupt the inflammatory cascade by working at both sides of the blood-brain barrier (the brain vascular endothelium and brain microglia and neuronal cells) to decrease brain

ABBREVIATIONS: ACN, acetonitrile; AD, Alzheimer's disease; Ang-(1-7), angiotensin-(1-7); Ang II, angiotensin II; A779, 5-L-isoleucine-7-D-alanine1-7-angiotensin II; ANOVA, analysis of variance; AUC, area under the curve; CCL, chemokine (C-C motif) ligand; CIPL, corrected integrated path length; CXC, chemokine (C-X-C motif); DAB, diaminobenzidine tetrahydrochloride; DADLE, D-alanine-D-leucine-enkephalin; DHE, dihydroethidium; $\mathrm{EF} \%$, percentage of ejection fraction; GCSF, granulocyte colony-stimulating factor; HF, heart failure; HUVEC, human umbilical vein endothelial cell; Iba-1, ionized calcium binding adaptor molecule 1; IL, interleukin; IS, internal standard; LC, liquid chromatography; LCA, left coronary artery; MasR, Mas receptor; MI, myocardial infarction; MIP, macrophage inflammatory protein; MS, mass spectrometry; MWM, Morris water maze; NOR, novel object recognition; PK, pharmacokinetic; PNA5, Ang-1-6-O-Ser-Glc- $\mathrm{NH}_{2}$; ROS, reactive oxygen species; ROI, region of interest; TFA, trifluoroacetic acid; TNF- $\alpha$, tumor necrosis factor- $\alpha$; $t_{1 / 2}$, half-life; VCID, vascular contributions to cognitive impairment and dementia. 
ROS production and neuroinflammation and to improve cerebral vascular blood flow while having minimal side effects.

The peptide angiotensin-(1-7) (Ang-1-7) meets these criteria. Ang-(1-7) is known to decrease brain ROS production and inflammation in preclinical models of HF (Hay et al., 2017), to increase cerebral blood flow (Zimmerman et al., 2002; Jiang et al., 2014), and to be safe in clinical studies in humans (Rodgers et al., 2006). Within the brain, the Mas receptor (MasR) is found on neurons, microglia, and vascular endothelial cells. Activation of MasR decreases ROS and brain inflammation, increases cerebral circulation via increases in endothelial nitric oxide release and inhibition of inflammatory cytokines, and inhibits hypoxia-inducing factor- $1 \alpha$ (Ferrario, 2006; Zheng et al., 2014; Chang et al., 2016; Salminen et al., 2017). Because the MasR is found in high quantities within the hippocampus and perirhinal cortex as well as in vascular endothelial cells, Ang-(1-7) is hypothesized to be effective in targeting memory impairments related to hypoxia and inflammation-related neurodegenerative disease (Hay et al., 2017).

In the present study, our team has taken a novel approach to combine our extensive experience improving peptide performance via glycosylation (Egleton et al., 2001; Lefever et al., 2015) with our experience with the G-protein-linked MasR and its agonist Ang-(1-7) (Mordwinkin et al., 2012; Forte et al., 2016; Hay et al., 2017; Nation et al., 2018). We have developed a series of novel glycosylated Ang-(1-7) MasR agonists with enhanced pharmacokinetic (PK)/pharmacodynamics properties. These agonists are designed to improve bioavailability, stability, and brain penetration of Ang-(1-7). Our research team has developed, optimized, and completed high-throughput in vitro and in vivo screens of novel synthetic glycopeptide derivatives of Ang-(1-7) that have outstanding brain penetration and enhanced stability (Dhanasekaran and Polt, 2005; Malakoutikhah et al., 2008; Jones and Polt, 2015). We have completed a full physiochemical and mechanistic preclinical study of the in vitro and in vivo behavioral properties of our lead candidate, Ang-1-6-O-Ser-Glc-NH $\mathrm{N}_{2}$ (PNA5). We hypothesize that this novel glycosylated Ang-(1-7) derivative will be a "first-in-class" treatment of inflammation-induced cognitive impairment.

The goals of the present study were to 1 ) describe the physiochemical properties of PNA5 in comparison with the native Ang-(1-7) peptide, 2) determine whether PNA5 might be a candidate therapeutic agent to reduce or reverse cognitive impairment induced by systemic inflammation, 3) determine whether PNA5 can inhibit ROS formation in a human cell line, and 4) begin to identify possible systemic biomarkers associated with PNA5 treatment. We used our previously described mouse model of VCID/HF-induced cognitive impairment (Hay et al., 2017) and hypothesized that sustained treatment with PNA5 will attenuate or reverse the VCID/HF-induced cognitive impairment.

\section{Materials and Methods}

\section{Chemicals and Reagents}

Chemicals were purchased through Sigma-Aldrich (St. Louis, MO), unless otherwise indicated. An internal standard (IS) [D-alanine-Dleucine-enkephalin (DADLE)] was purchased through American Peptide Company, Inc. (Sunnyvale, CA).
Synthesis of Peptides. Glycosylated peptides were synthesized by the Polt Laboratory (University of Arizona) via solid-phase synthesis, using methods previously described (Lowery et al., 2007; Jones and Polt, 2015; Lefever et al., 2015).

In Vitro Stability of Angiotensin Derivatives. Degradation studies were performed in rat serum in a $37^{\circ} \mathrm{C}$ water bath as previously described (Mabrouk et al., 2012; Heien, 2015). Peptides were spiked for a final concentration of $\sim 100 \mu \mathrm{M}$, and time points were determined by apparent degradation rate after $n=2$ trials. At each time point, $10 \mu \mathrm{l}$ of rat serum + peptide was removed and spiked with $1 \mu \mathrm{l}$ of glacial acetic acid to quench peptidase activity, $1 \mu \mathrm{l}$ of $100 \mu \mathrm{M}$ DADLE as an IS, and $1 \mu \mathrm{l}$ of $25 \mathrm{mM}$ octyl sulfonate as a pairing agent. Samples were desalted using $\mu$ - $\mathrm{C}_{18}$ Zip Tips (EMD Millipore, Burlington, MA) according to the following protocol, on a 10- $\mu$ l scale: three conditioning steps with acetonitrile (ACN), followed by three conditioning steps with $0.1 \%$ trifluoroacetic acid (TFA) in nanopure water (v/v). Ang-(1-7) derivatives were found to bind best with 15 binding steps. A single wash with $0.1 \%$ TFA in water desalted the column, and the procedure was completed with 10 elution steps in a 60:40 can $/ \mathrm{H}_{2} \mathrm{O}: 0.1 \% \mathrm{TFA}(\mathrm{v} / \mathrm{v})$ solution. Samples were then brought to $100 \mu \mathrm{l}$ in $50: 50 \mathrm{can} / \mathrm{H}_{2} \mathrm{O}(\mathrm{v} / \mathrm{v})$ solution with $0.1 \%$ formic acid for electrospray compatibility. Samples were analyzed using tandem mass spectrometry (MS) on an AB Sciex QStar Elite Mass Spectrometer (Applied Biosystems, Foster City, CA) with a $20-\mu \mathrm{l}$ injection loop. Samples were run in duplicate, averaged for each time point, and normalized to the 0 -minute time point as the 100\% signal. Analyses were performed in GraphPad Prism 5 (GraphPad Software, La Jolla, CA), and degradation trials with exponential fits poorer than $r^{2}<0.7$ were discarded.

\section{Animals and Surgical Procedures}

Animals for Dialysis. Male Sprague-Dawley rats (weight, $275-325 \mathrm{~g}$ ) were used for dialysis experiments in vivo. Animals were purchased from Envigo Laboratories (Indianapolis, IN) and housed in a temperature- and humidity-controlled room with 12-hour reversed light/dark cycles and with food and water available ad libitum. All animals were treated as approved by the Institutional Animal Care and Use Committee at the University of Arizona and in accordance with the National Institutes of Health Guidelines for the Care and Use of Laboratory Animals. Both the number of animals used and their suffering were minimized. The number of animals needed for each experiment was determined using a $\mathrm{G}^{*}$ Power analysis (Faul and Coronado, 2015).

Carotid Artery Catheterization. Catheterized animals were purchased directly from Envigo Laboratories. At their facility, animals were anesthetized with $1.5 \%-2 \%$ isoflurane mixed in medical grade oxygen $(1.5 \mathrm{l} / \mathrm{min})$ and the following two incisions were made: 1) a $0.5-\mathrm{cm}$ midline incision between the scapulae; and 2) a $2.0-\mathrm{cm}$ incision to the right of the midline of the ventral neck. The right carotid artery was isolated, and two 4-0 silk sutures were placed at the caudal and cranial ends of the vessel. The cranial suture was immediately tied off and a bulldog clamp was placed above the caudal tie to temporarily occlude blood flow. A carotid venotomy was performed, and a piece of PE-10 catheter tubing, preflushed with a heparin/glycerol solution (500 IU heparin/1 ml; 50:50 ratio), was passed through the vessel toward the heart. The bulldog clamp was then released, and the caudal suture was tied off to secure the catheter in place. The catheter was then threaded through a subcutaneous pocket and connected to a vascular access port (Instech Laboratories, Plymouth Meeting, PA) at the scapular incision. The ventral neck incision was closed with stainless steel wound clips, whereas the vascular access port was secured and the scapular incision was closed with 4-0 silk sutures. Animals were monitored during recovery for 3-4 days before being shipped to University of Arizona facilities. Catheter patency was evaluated within the first 24 hours of arrival and subsequently every $2-3$ days to ensure patency prior to microdialysis. 
Microdialysis Surgery. Upon arrival at our facility, animals were acclimated for 5-7 days. Procedures for microdialysis have been described previously (Mabrouk et al., 2012; Heien, 2015). Animals were anesthetized with $1.5 \%-2 \%$ isoflurane mixed in medical grade $\mathrm{O}_{2}$ (1.5 $\mathrm{l} / \mathrm{min}$ ) and positioned inside a stereotaxic frame (David Kopf Instruments, Tujunga, CA). A midsagittal incision was made along the skull, and a CMA 11 guide cannula (CMA Microdialysis, Kista, Sweden) was implanted in the striatum at the following coordinates: anteroposterior $+1.0 \mathrm{~mm}$ and mediolateral $+3.2 \mathrm{~mm}$ relative to bregma; and dorsoventral $-3.4 \mathrm{~mm}$ from the surface of the brain. The guide was then replaced with a CMA 11 probe containing a 4.5-mm cuprophane membrane with a molecular weight cutoff of $6 \mathrm{kDa}$. After the probe was positioned, the animals remained under anesthesia for the remainder of the experiment.

Microdialysis and Blood Draws. To directly compare the in vivo lifetime and blood-brain barrier penetration of both Ang-(1-7) and PNA5, we developed a method that involves simultaneous blood draws and microdialysis (Mabrouk et al., 2012; Heien, 2015). The compounds were injected intravenously at $10 \mathrm{mg} / \mathrm{kg}$ via a single tail vein injection. Blood draws were taken at $t=-10,1,5,10,20,30,40,50,60,70,80$, and 90 minutes (where $t=0$ corresponds to injection). Microdialysis fractions were time locked with blood draws at 10-minute increments such that blood draws correlated with the median time of the microdialysis fraction. For microdialysis, a dual-syringe pump (PHD 2000; Harvard Apparatus, Holliston, MA) was set to a flow rate of $0.5 \mu \mathrm{l} / \mathrm{min}$. Samples were collected and preserved in a solution containing $10 \% \mathrm{v} / \mathrm{v}$ acetic acid, $2 \% \mathrm{v} / \mathrm{v}$ ACN, and $140 \mathrm{pM}$ DADLE. DADLE was used as an IS. Baseline blood $(\sim 100 \mu \mathrm{l})$ and dialysate samples were collected. Subsequent time-locked samples (with blood being drawn at the median of the dialysate time range) were then collected every 10 minutes for 90 minutes, after a tail vein injection at $t=0$ minute. In addition, blood samples were also collected at both 1 and 5 minutes postinjection. CSF samples were immediately frozen on dry ice. Blood samples were centrifuged to separate serum from the red blood cells. Serum was drawn off, and $10 \mu \mathrm{l}$ of serum was spiked with glacial acetic acid to quench peptidase activity, and DADLE was added to a final concentration of $5 \%$ glacial acetic acid and $140 \mathrm{pM}$ IS before being frozen on dry ice. Postexperiment, samples were stored in a $-80^{\circ} \mathrm{C}$ freezer until analysis.

All samples were desalted using $\mu-\mathrm{C}_{18}$ Zip Tips (EMD Millipore) according to the protocol above, yielding a final solution composed of $10 \mu \mathrm{l}$ of $60: 40 \mathrm{ACN} / \mathrm{H}_{2} \mathrm{O} / 0.1 \% \mathrm{TFA}(\mathrm{v} / \mathrm{v})$. For reverse-phase liquid chromatography (LC), samples should be dissolved in aqueous solution; thus, samples were vacuum centrifuged until $1 \mu \mathrm{l}$ of solution remained and were reconstituted in $5 \mu \mathrm{l}$ of $\mathrm{H}_{2} \mathrm{O}$ with $0.1 \% \mathrm{TFA}(\mathrm{v} / \mathrm{v})$. Samples $(5 \mu \mathrm{l})$ were injected onto the column and separated using a gradient elution. Target compounds were quantified by summing the height of previously identified fragment peaks in the LC-MS ${ }^{3}$ spectra. The serum and CSF concentrations were estimated using a calibration curve, accounting for the dilution factors and the probe recoveries for each experiment. Standards were matrix matched to samples and underwent the same sample preparation steps.

Data Analysis. All data are presented as the mean \pm S.E.M. The $C_{\max }$ and the area under the curve (AUC) were calculated for both Ang-(1-7) and PNA5. Data are calculated as the log fold change for each animal tested $(n=8)$.

\section{Behavior Studies}

Animal Housing. Adult C57BL/6 male mice $(N=27)$ (Envigo Laboratories; animals arrived in the facility at 2 months of age and were maintained for 3 months) were used in the behavior studies. Two to four mice were housed together according to their assigned experimental group in cage racks that were temperature controlled and on a 12-hour light/dark cycle. All mice had had ad libitum access to food and water for the duration of the experiments. All experiments were performed with adherence to guidelines approved by the Institutional Animal Care and Use Committee at the University of
Arizona and to the 2012 National Institutes of Health Guidelines for the Care and Use of Laboratory Animals.

Animal Groups. Mice were assigned randomly to either the control [sham myocardial infarction (MI) surgery + saline] $(n=6)$, HF-treated (HF-PNA5, $n=10$ ), or HF-saline (HF-saline, $n=11$ ) group. Animal numbers were based on a power analysis for statistical significance. Eight weeks after MI surgery, animals received 21 days of daily morning subcutaneous injections of either saline or PNA5 $(1.0 \mathrm{mg} / \mathrm{kg}$ dissolved in saline). The animals were subjected to the novel object recognition (NOR) test and spatial memory Morris water maze test, respectively, 3 and 7 days after the final injection.

Mouse Model of MI. Prior to surgery, the body weight of the mice was obtained, and they were anesthetized with $2.5 \%$ isoflurane in a mixture of air and $\mathrm{O}_{2}$. The induction of MI was performed via left coronary artery (LCA) ligation as described by Gao et al. (2000). To induce the MI, a thoracotomy was performed on the left side through the fourth intercostal space and the LCA was ligated permanently. Confirmation of the occlusion of the LCA was obtained by observing a whitening slight change in color of the anterior wall of the left ventricle downstream of the ligature. For the control animals, a sham surgery was performed, and mice underwent the same procedure with the exception of ligating the LCA. To assess ventricular function, cardiac morphometry, and remodeling, echocardiography was performed (as above) at 1, 4, 8, and 12 weeks postsurgery.

\section{Echocardiography}

Echocardiography was performed before MI surgery and then repeated at $1,4,8$, and 12 weeks post-MI using a Vevo 2100 HighResolution Imaging System (Visual Sonics, Toronto, ON, Canada) and a $25-\mathrm{MHz}$ transducer. The chest hair of the animals was removed using a chemical depilatory. To examine cardiac function at physiologically relevant heart rates (Kass et al., 1998) and to eliminate any anesthesia effects, the echocardiograms were performed in awake mice. Two-dimensional M-mode echocardiographic images were obtained from the parasternal short-axis view at the level of the midventricle. The thickness of the left ventricular wall and cardiac chamber dimensions were measured. The interventricular septum, left ventricular posterior wall thickness, and left ventricular internal dimension were measured from the M-mode images. Data were analyzed off-line using Vevo 2100 analytic software (Visual Sonics). The data were obtained in triplicate and averaged.

NOR. The texting box was an evenly illuminated Plexiglas container $(12 \times 12 \times 12 \mathrm{~cm})$ placed on a table inside a sound-isolated observation room. All walls of the box were blackened, and the floor was gray with a grid that was used to ensure that the placement of the objects did not change between phases of the test. A digital camera was used to record mouse behavior and exploration of the object. The digital image from the camera was fed into a computer in the adjacent room. The time the mice spent exploring the objects was measured using digital stopwatches. All examiners were blinded to the treatment groups. All data were downloaded to Excel (Microsoft, Redmond, WA) files for analysis. Three sets of distinct objects were used for the test.

Procedure. The three phases of the NOR task included a habituation phase, a familiarization phase, and a test phase. During the habituation phase, on the first and second day, mice were brought to the observation room and habituated for 10 minutes per day to the empty box. On day 3, the "familiarization" trial was performed with each mouse. This trial consists of two identical objects followed by a preset delay period and then a "test" trial in which one object was novel and the second object was identical to the one in the familiarization phase. All objects were available in triplicate copies of each other so that no object needed to be presented twice. Objects were composed of plastic, glass, or wood that varied in size, color, and shape. For each phase, each mouse was placed into the box the same way, oriented to the center of the wall opposite to the objects. To avoid the possibility of olfactory cues, the box and objects were well cleaned with $70 \%$ ethanol 
and towel dried after each trial. For the familiarization phase, mice were given the opportunity to explore the two identical objects for 4 minutes and then they were returned to their original cages. The "test phase" began after a 2-hour delay. The mice were placed back in the testing box, where one of the two identical objects presented in the familiarization phase was changed to a novel object, and the mouse was given the opportunity to explore these objects for another 4 minutes. To measure the exploratory behavior, the time spent exploring each object was assessed as the animal orienting its nose toward the object at a distance of $\sim 2 \mathrm{~cm}$ or less (Ennaceur and Delacour, 1988). Other behaviors, such as rising up on the object or resting against the object was not considered to be exploratory behavior. The scoring of exploration was blinded and made by an observer who was unaware of the experimental group of each mouse (HF vs. sham) and treatment group.

Analysis. To determine the discrimination ratio (DRatio), the time spent exploring the novel object ( $t$ novel) minus the time spent exploring the familiar object ( $t$ familiar) during the test phase divided by the total exploration time was calculated, as follows: DRatio = $(t$ novel $-t$ familiar $) /(t$ novel $+t$ familiar $)$. Data were analyzed from first 2 minutes of the "test phase." A score that was positive indicates more time spent with the novel object, a score that was negative indicates more time spent with the familiar object, and a zero score indicates a null preference. All of the data from the NOR test were analyzed using one-way analysis of variance (ANOVA), between subjects (ANOVA). Differences between individual groups were tested using the post hoc Tukey honestly significant difference test. In comparisons between groups of different sample sizes, equal variance was tested using a modified Levene's test. We used Microsoft Excel with Daniel's XL Toolbox for all statistical tests, and $P$ values and $\alpha$ levels was set at the 0.05 level. Error bars represent the S.E.M.

Morris Water Task: Spatial Learning and Memory/Visual Test. The Morris water maze was constructed using was a large circular pool approximately $1.5 \mathrm{~m}$ in diameter, filled with water at $25^{\circ} \mathrm{C}$. The water was rendered opaque by adding white, nontoxic Crayola paint. Just below the surface of the water, an escape platform was hidden. On the walls surrounding the pool, high contrast visual cues were fixed. To record the progress of the task, a digital camera was suspended over the pool and connected to a computer in the observation room.

Procedure. For the spatial version of the Morris water task, the mice were had six training trials per day which lasted for 4 consecutive days. For these trials, an escape platform was hidden below the surface of water. Seven different start locations around the perimeter of the pool were predetermined, and each animal performed two successive trials before the next mouse was tested. The order of the release locations was pseudo-randomized for each mouse such that no mouse was released from the same location on two consecutive trials. The day after the 24 spatial trials, the mice performed a probe trial in which the platform was removed, and a mouse swam in the pool for 60 seconds. After the probe trial, the mice were screened for visual acuity where the escape platform was raised above the surface of the water, but the position of the platform changed between each trial. Performance on the swim task was analyzed with a commercial software application (ANY-maze, Wood Dale, IL). Because different release locations produce variability in the latency to reach the escape platform, a corrected integrated path length (CIPL) was calculated to ensure the comparability of performances of mice across different release locations. The CIPL value measures the cumulative distance over the time from the escape platform corrected by the swimming velocity of an animal and is equivalent to the cumulative search error described by Gallagher et al. (1993). Therefore, regardless of the release location, if the mouse swims mostly toward the escape platform, the CIPL value will be low. In contrast, the more time a mouse spends swimming in directions away from the platform, the higher the CIPL value.

Analysis. The CIPL was used as the primary measure of spatial learning. The data from the Morris water task was analyzed using a
Welch $t$ test with individuals performing the calculations $r$ blinded to the surgical group (HF vs. control) and treatment group of the mouse. All statistical tests and $P$ values were calculated using Microsoft Excel with Daniel's XL Toolbox, and the $\alpha$ was set at the 0.05 level. Error bars represent the S.E.M.

Histology. After euthanasia, hearts were quickly extracted, washed, and immersed in 10\% neutral buffered formalin for 24 hours of fixation before histologic evaluation. The fixed hearts were processed, embedded in paraffin, sectioned, and stained with H\&E.

Analysis. Differences over time within each group were determined by a repeated-measures ANOVA. Mean differences between different groups were analyzed using two-way ANOVA (Prism; GraphPad Software). Post hoc tests were carried out using Bonferroni $t$ tests.

\section{Human Endothelial Cell Culture and ROS Measurements}

Cell Culture. Human umbilical vein endothelial cells (HUVEC) were purchased from American Type Culture Collection (Manassas, VA). The cells were cultured in media consisting of modified Kaighn's modification of Ham's F-12 medium (American Type Culture Collection) supplemented with 10\% FBS (American Type Culture Collection), $0.1 \mathrm{mg} / \mathrm{ml}$ heparin (Sigma-Aldrich), $0.03 \mathrm{mg} / \mathrm{ml}$ endothelial cell growth supplement (Upstate, Lake Placid, NY), $100 \mathrm{U} / \mathrm{ml}$ penicillin, and $100 \mathrm{~g} / \mathrm{ml}$ streptomycin in a humidified chamber at $37^{\circ} \mathrm{C}$ in a $5 \%$ $\mathrm{CO}_{2} / 95 \%$ air atmosphere. The cells were cultured in T75 flasks and 12 -well tissue culture plates. For these studies, the cells were used for up to eight passages. Two days before the experiment, cells were plated onto $12-\mathrm{mm}$ culture slips treated with poly D-lysine.

ROS Measurements. The measurement of ROS generation was determined using real-time fluorescence microscopy with the measurement of conversion of the fluorescent probe dihydroethidium (DHE) to ethidium (Sigma-Aldrich). DHE is cell permeable and provides nuclear fluorescence at an excitation wavelength of $520 \mathrm{~nm}$ and an emission wavelength of $610 \mathrm{~nm}$, an indicator after reaction with superoxide radicals to form ethidium that in turn interacts with DNA. We used an Olympus (Tokyo, Japan) FV500 confocal microscope system equipped with a green HeNe laser for excitation and a longpass red filter to detect ethidium fluorescence. Laser intensity and gain were identical for all images.

Analysis. Imaging data were generated from an Olympus FV500 microscope and then analyzed by NIH ImageJ. Fluorescent cells were identified as regions of interest (ROIs) using a standardized ImageJ ROI manager. From the identified ROIs, the integrated density measurements were taken. Group differences were analyzed by using a Student's $t$ test. All statistical tests and $P$ values were calculated using Microsoft Excel with Daniel's XL Toolbox, and $\alpha$ was set at the 0.05 level. Error bars represent the S.E.M.

\section{Multiplex Immunoassay: Early Disease, 1-Week Ang-(1-7) Treatment}

To determine the early inflammatory response during the progression of $\mathrm{HF}$ and the early effects of 1 week of PNA5 treatment, we used a MILLIPLEX MAP Mouse Cytokine/Chemokine Magnetic Bead PanelImmunology Multiplex Assay (MCYTOMAG-70K; Millipore Sigma, Burlington, MA) for quantifying inflammatory markers in animals 2 weeks post-MI and 1 week after PNA5 treatment. These animals were not tested for changes in cognition. C57BL/6 male mice ( 3 months old) were subjected to MI (permanent ligation of the left coronary as previous) or sham surgery. One week post-MI or control, mice were administered ( $1 \mathrm{mg} / \mathrm{kg}$ ) PNA5 or saline by injection (subcutaneous) for 1 week (three groups, $n=8$ each). Two weeks post-MI, blood was collected in murine EDTA tubes, centrifuged to obtain plasma, and rapidly frozen in liquid nitrogen. Plasma cytokines, chemokines, and additional inflammatory analytes were detected and quantified by multiplex immunoassay using a MAGPIX Multiplexing Instrument and accompanying Multiplex Analyst software (EMD Millipore). 
This assay allows for the simultaneous measurement of the following cytokines: granulocyte colony-stimulating factor (GCSF), granulocytemacrophage colony-stimulating factor, interferon $\gamma$, interleukin (IL)1a, IL-1B, IL-2, IL-4, IL-5, IL-6, IL-7, IL-9, IL-10, IL-12 (p40), IL-12 (p70), IL-13, IL-15, IL-17, interferon $\gamma$-induced protein 10, chemokine (C-X-C motif) (CXC) ligand 1 (KC), chemokine (C-C motif) ligand (CCL) 2 (monocyte chemoattractant protein-1), CCL3 [macrophage inflammatory protein (MIP) $1 \alpha$ ], CCL4 (MIP1 $\beta$ ), CXC ligand 2 (MIP2), CCL5 [RANTES (regulated on activation normal T cell expressed and secreted)], and tumor necrosis factor- $\alpha$ (TNF- $\alpha$ ). Differences between groups were analyzed by using a Student's $t$ test. All statistical tests and $P$ values were calculated using Microsoft Excel with Daniel's XL Toolbox, and $\alpha$ was set at the 0.05 level. Error bars represent the S.E.M.

\section{Immunohistochemistry}

On the day after the final water maze task and 8 days after the last injection of PNA5, animals were euthanized via $\mathrm{CO}_{2}$, and brain tissue was removed and immersed in $4 \%$ paraformaldehyde, $\mathrm{pH} 7.4$, for 7 days and then changed to $30 \%$ sucrose in PBS for cryoprotection. Brains were then frozen at $-20^{\circ} \mathrm{C}$ until processed. Fixed brains were coronally blocked between -4 and $0 \mathrm{~mm}$ from bregma. Dry ice and Tissue Freezing Media (TFM-5; Triangle Biomedical Science, Inc., Durham, NC) were used to freeze tissue blocks onto a stage that was mounted onto a sliding microtome. The frozen brains were serially coronally sectioned $(30 \mu \mathrm{m})$ using the sliding microtome into six-well tissue culture plates containing PBS with $0.02 \%$ sodium azide for later immunostaining. Sections were allowed to free float and were permeabilized with $0.3 \% \mathrm{H}_{2} \mathrm{O}_{2}$ for 1 hour, blocked with $5 \%$ donkey serum with $0.3 \%$ Triton $\mathrm{X}-100$ for 2 hours, and incubated with primary Iba-1 (ionized calcium binding adaptor molecule 1) antibody for 48 hours (goat, 1:200; catalog number ab5076; abcam, Cambridge, MA). Negative controls for the immunostaining consisted of equivalent sections subjected to the same protocol but omitting the primary antibody. Sections were incubated with biotinylated anti-goat IgG (1: 300; BA-100; Vector Laboratories, Burlingame, CA) followed by ABC complex for 30 minutes (Vectastain Elite Kit; Vector Laboratories) and reaction with diaminobenzidine tetrahydrochloride (DAB) (SigmaAldrich) for 5-15 minutes in Tris, $\mathrm{pH}$ 7.7. Sections were washed multiple times, mounted onto gelatin-coated slides, allowed to air dry overnight, and coverslipped for analysis.

\section{Image Analyses and Quantitative Assessment of Immunostaining}

For quantitative assessment, measurements of immunoreactivity within the meningeal layer of the cortical surface were obtained in brains from HF-PNA5, HF-saline, and control animals. Ten ROIs of the cortical surface/meningeal sections were randomly identified in two brains from each group for a total of 20 ROIs per group. Images for analysis were taken using a $10 \times$ objective on an Olympus Imt-2 Inverted Phase Contrast Microscope fitted with an Olympus HD digital camera. Camera exposure settings were identical for all images. The level of DAB staining was quantified with NIH ImageJ software. The ROI manager in ImageJ was used to create a standardsized ROI, and this same size ROI was used to analyze each slice. Identical image acquisition settings were used for all images. To ensure a uniform background across sections, we use ImageJ background subtraction prior to thresholding the images. We used an identical standard ImageJ thresholding tool setting for each image acquired. These image acquisition control techniques allowed us to successfully quantify staining that was duplicated in every image that was compared. From the threshold image, integrated density measurements from each ROI were obtained. Differences between groups were analyzed using a Student's $t$ test. All statistical tests and $P$ values were calculated using Microsoft Excel with Daniel's XL Toolbox, and $\alpha$ was set at the 0.05 level. Data are presented as the mean, with error bars representing the S.E.M.

\section{Results}

Glycosylated Ang-(1-7) has Increased In Vitro Lifetime. As illustrated in Fig. 1A, glycosylation of Ang-(1-7) (DRVYIHP) replaces the seventh residue (proline) with a serine, and has a glucose sugar moiety attached to the serine and is amidated on the $\mathrm{C}$ terminus, resulting in PNA5. The molecular weight of PNA5 is $1278 \mathrm{~g} / \mathrm{mol}$. Figure $1, \mathrm{~B}$ and C, illustrates the in vitro degradation of Ang-(1-7) and PNA5, and the exponential fit curves from mean values for 0-60 minutes for both Ang-(1-7) $\left(r^{2}=0.95\right)$ and PNA5 $\left(r^{2}=0.6\right)$ are reported as mean \pm S.E.M. $(n=8)$. After 20 minutes, PNA5 has $89.6 \% \pm 7.9 \%$ remaining versus Ang-(1-7) has $6.6 \% \pm$ $7.9 \%$ remaining ( $* P<0.05$, PNA5, $n=8$; Ang-(1-7), $n=6$; $F=18.62, P=0.002$, one-way ANOVA, Bonferroni post hoc test). The glucoside analog PNA5 (AT1-6-Ser(OGlc)- $\mathrm{NH}_{2}$ ) shows increased serum half-life $\left(t_{1 / 2}\right)$ of $1.0 \pm 0.2$ hour compared with the native Ang-(1-7) of $0.25 \pm 0.05$ hour.

Glycosylated Ang (1-7) Degradation Analysis. Importantly, the degradation analysis of PNA5 (Fig. 2) shows that the primary metabolites are the initial cleavage of the glucose followed by subsequent degradation at the Ang-(1-6) six peptide cleavage sites. Figure $2 \mathrm{~A}$ illustrates the fragmentation pattern for using LC-MS and $\mathrm{MS}^{3}$ spectra. The targeted analysis allows for low concentrations of peptide to be measured (Fig. 2B). The early and largest secondary fragments are Ang-(1-7) "like" with a very similar structure to the native Ang-(1-7) peptide. We saw no evidence that the modification via glycosylation yielded harmful degradation products or products that are not produced by the natural degradation of endogenous Ang-(1-7).

Comparison of PNA5 to Native Ang-(1-7) In Vivo. Given the physiochemical changes that glycosylation affords to the Ang-(1-7) peptide, we hypothesized that we would observe improved peptide bioavailability in the circulation, as well as improved concentrations in the brain. As illustrated in Fig. 3, we measured both serum and CSF levels of both PNA5 and Ang-(1-7) after $10 \mathrm{mg} / \mathrm{kg}$, i.v., injection in healthy rats. In serum, PNA5 showed an average $C_{\max }$ and AUC log fold increase of $2.6 \pm 0.1$ and $2.4 \pm 0.1$ over Ang-(1-7) (Fig. 3C), respectively $(n=8)$. In CSF, CSF showed an average $C_{\max }$ and AUC $\log$ fold increase of $2.7 \pm 0.2$ and $2.3 \pm 0.1$ over Ang-(1-7) (Fig. 3F), respectively $(n=8)$.

Table 1 is a summary of the physiochemical characteristics of PNA5 compared with Ang-(1-7).

Cardiac Assessment and Behavioral Testing. Our previously published studies with the native Ang-(1-7) peptide (Hay et al., 2017) showed that 3 weeks of treatment with Ang-(1-7) protected and reversed the cognitive impairment observed in our model of inflammation-induced vascular dementia in VCID/HF. We hypothesized in the present study that PNA5 would be similar to, if not better than, Ang-(1-7) at protecting from VCID/HF-induced cognitive impairment.

The time course for the behavioral experiments is illustrated in Fig. 4A. Mice were assigned to groups either HF-PNA5 $(n=11)$, HF-saline $(n=10)$, and control $(n=6)$ 7 days prior to any interventions. The $\mathrm{HF}$ mice then underwent coronary artery ligation and controls received a sham surgery where the heart was exposed but the coronary artery was not ligated. Heart function was audited by conscious echocardiography every 4 weeks. On day 56 , PNA5 $(1.0 \mathrm{mg} / \mathrm{kg})$ or saline subcutaneous daily injections began and continued 
A

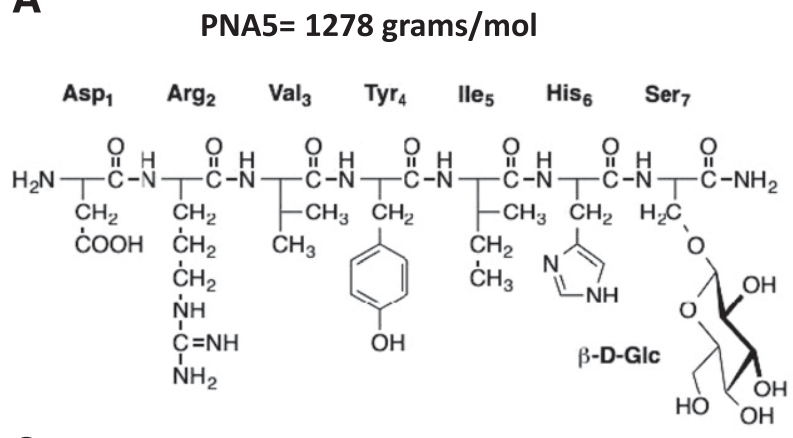

C

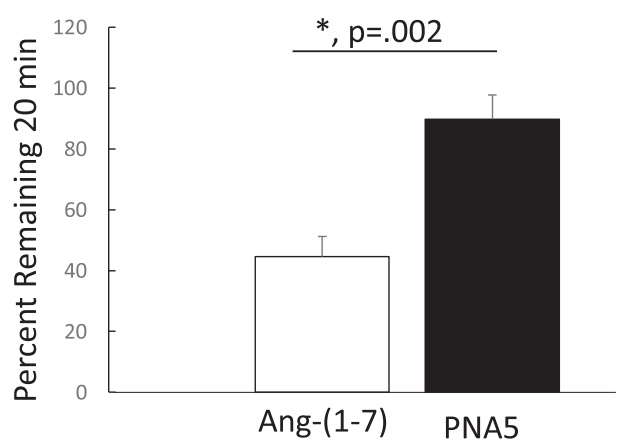

B

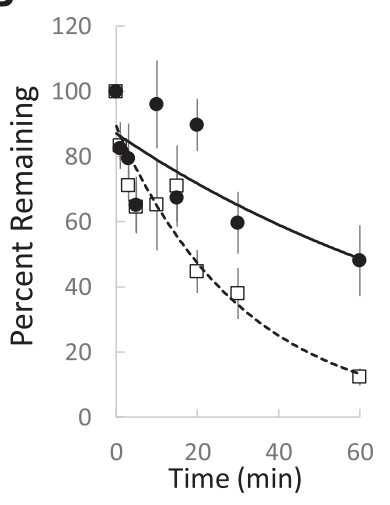

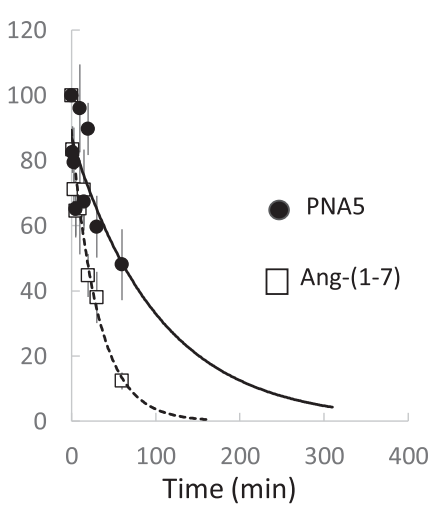

\begin{tabular}{|l|l|}
\hline Compound & $\mathbf{t}_{1 / 2}(\mathrm{hr})$ \\
\hline Native Ang 1-7 & $0.25+0.05$ \\
\hline PNA5 & $1.06+0.20$ \\
\hline
\end{tabular}

Fig. 1. Structure and in vitro $t_{1 / 2}$ of PNA5. (A) Glycosylation of Ang-(1-7) (DRVYIHP) replaces the seventh residue (proline) with a serine and has a glucose sugar moiety attached to the serine and is amidated on the $\mathrm{C}$ terminus, resulting in PNA5. (B) In vitro degradation of Ang-(1-7) and PNA5. Left: The exponential fit curves from mean values for 0-60 minutes for both Ang-(1-7) $\left(r^{2}=0.95\right)$ and PNA5 $\left(r^{2}=0.6\right)$ reported as the mean \pm S.E.M. $(n=8)$. Right: Extrapolated time scale of 0-300 minutes. (C) After 20 minutes, PNA5 has $89.6 \% \pm 7.9 \%$ remaining vs. Ang-(1-7) with $6.6 \%+7.9 \%$ remaining. $(* P<0.05$; PNA5, $n=8$; Ang-(1-7), $n=6 ; F=18.62, P=0.002$, one-way ANOVA, Bonferroni post hoc test.

until day 77. Three days after the last injection, the NOR test began, and on day 87, a full week after the last PNA5 treatment injection, spatial memory [Morris water maze (MWM)] began and was completed 11 days after the final PNA5 injection. Immediately after conclusion of the MWM, animals were anesthetized and sacrificed, and brains were processed.

Ventricular Remodeling and Echocardiography. To examine the pathology of the hearts at the completion of the 12 -week study period, hearts were removed, fixed, and stained with H\&E (see Materials and Methods). Representative sections of H\&E-stained hearts are illustrated in Fig. 4B for control and HF-saline-treated hearts. The myocardium from the mice that underwent MI exhibited extensive loss of myocardium within the infarcted segment compared with control mice. This pattern of wall thinning was evident in all MI-operated mice, independent of PNA5 or saline treatment.

To measure the progression of change in cardiac function, we performed serial echocardiography at $1,4,8$, and 12 weeks post-MI in the HF mice to correlate the level of cardiac dysfunction with the cognitive impairment. Figure 4C illustrates representative M-mode echocardiographic images from each experimental group. The changes in the dimensions of the ventricular chamber after MI impacted cardiac function, as evidenced by the decrease in cardiac function [percentage ejection fraction (EF\%)] (Fig. 4D). By week 1 post-MI, the EF\% values of the HF-saline and HF-PNA5 groups were significantly different from baseline and different from control (week 1: HF-saline, $37.4 \pm 3 \%$; HF-PNA5, $36.4 \pm 2 \%$; control, $56.4 \pm$ $2 \%$; ${ }^{*}><0.05$ ). The EF\% continued to decline in both HF-saline and HF-PNA5, both reaching $22.6 \%$ by week 12 . From week 1 to 12, the EF\% values for HF-saline, HF-PNA5, and control mice continued to be significantly different. PNA5 treatment did not affect pattern wall thinning or EF.

Effect of PNA5 Treatment on NOR Performance. We used the NOR test, which is designed to test the ability of an animal to distinguish between familiar and novel objects, to determine changes in object recognition memory (Antunes and Biala, 2012). In the present study, the NOR test was given to all treatment groups 3 days after the last injection of PNA5.

The results of the NOR test in HF-PNA5 $(n=11)$, HF-saline $(n=10)$, and control $(n=6)$ mice are illustrated in Fig. 5A. Mean discrimination ratios, taken from the first 2 minutes of the "test phase," of HF and control mice after 3 weeks of treatment with PNA5 and 3 days after the last injection. A positive score indicates more time spent with the novel object, whereas a negative score indicates more time spent with the familiar object. A zero score indicates a null preference. PNA5 rescued object recognition performance. HF-PNA5 mice $(n=11)$ NOR discrimination ratios were similar to those of controls $(n=6)$ and were significantly greater than those of the HF-saline mice $(n=10)(+0.38 \pm 0.1$ vs. $-0.05 \pm 0.09, F(3,28)=8.13, P=0.0004$, ANOVA; $* P<0.05)$. These results demonstrate that treatment with PNA5 attenuates and even rescues object recognition memory impairment in our mouse model of VCID/HF-induced cognitive impairment.

To evaluate whether the differences in the results of the NOR test between the HF mice treated with saline versus PNA5 were related to differences in the exploration strategies between groups, we analyzed the total time the mice spent exploring each of the identical objects during the "familiarization phase" of the NOR test. During the familiarization phase, the DRatio between two identical objects would be expected to be near zero, and these data would be expected to 


\section{PNA5 DEGRADATION PATHWAY}

A
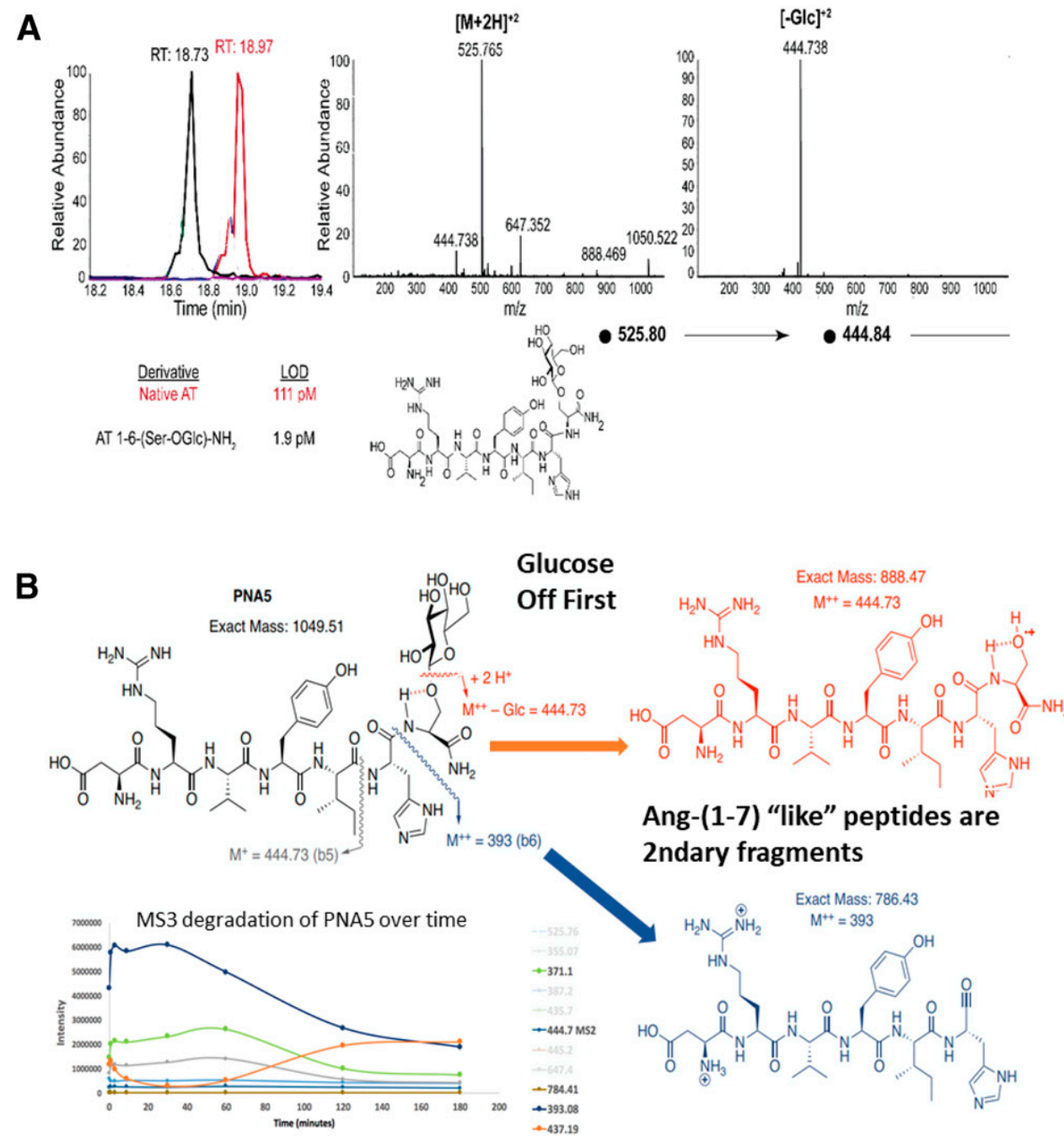

Fig. 2. PNA5 degradation pathway. (A) LC-MS and $\mathrm{MS}^{3}$ spectra for PNA5. The fragmentation pattern for PNA5 was determined, and an analysis was developed using LC-MS and $\mathrm{MS}^{3}$ spectra. This method of targeted analysis allows for low concentrations of peptide to be measured. A specific mass was selected and fragmented, and then another specific mass is selected. The limits-of-detection ranged from $1 \mathrm{pM}$ to $1 \mathrm{nM}$ for PNA5, depending on the specific fragmentation. Fragmentation of the $[\mathrm{M}$ $+2 \mathrm{H}]^{+2}$ parent peak of this glycosylated peptide gives one fragment in the $\mathrm{MS}^{2}$, which is loss of the sugar moiety with high efficiency. (B) PNA5 fragmentation begins with early removal of the carbohydrate (sugar) followed by subsequent degradation at the six peptide cleavage sites. The early largest secondary fragments are Ang-(1-7) like with very similar structure to the native Ang-(1-7) peptide. We saw no evidence that the modification via glycosylation yielded harmful degradation products or products that are not produced by the natural degradation of endogenous Ang-(1-7). be significantly different from the DRatio observed during the test phase when the mice were presented with both a familiar and novel object. As illustrated in Fig. 5B, the DRatio obtained during the familiarization phase is near zero in all three groups, suggesting that there is no difference in exploration patterns between the groups.

Effect of PNA5 Treatment on Spatial Memory and MWM Task Performance. We used the MWM task to examine changes in spatial memory. The MWM task is designed to test spatial learning, memory, and cognitive flexibility (Moser et al., 1995), In the present study, the MWM task was completed 10 days after the last injection of PNA5. As seen in Fig. 6A, PNA5 treatment improves spatial memory performance in HF mice. The HF-PNA5 mice $(n=11)$ showed significant improvement in spatial memory on day 3 of the swim task compared with HF-saline mice $(n=10)$ (HF-PNA5 mice, $23.5 \pm 3.2$; HF-saline mice, $33.0 \pm 3.6 ; F(1,123)=9.8, P=$ $0.002,{ }^{*} P<0.05$; one-way ANOVA, Bonferroni post hoc test).

To determine whether treatment with PNA5 improved memory persistence, we measured the daily savings score in all groups. The daily savings illustrates the memory persistence between subsequent trial days during the acquisition phase. The daily savings score was calculated as the CIPL score from the previous day minus the CIPL score from the subsequent day. As seen in Fig. $6 \mathrm{~B}$, PNA5 treatment $(n=11)$ significantly increased the daily savings score compared with saline alone $(n=10)$ and was similar to that of controls $(n=6$; day 1-day 2: HF-PNA5 mice, $8.0 \pm 0.9$; HF-saline mice, $4.4 \pm$ $0.6 ; F(1,117)=11.1, P=0.001$, one-way ANOVA, ${ }^{*} P<0.05$; day 2-day 3: HF-PNA5 mice, $6.7 \pm 0.5$; HF-saline mice, -1.7 $\pm 0.3 ; F(1,123)=168.8, P=7.8 \mathrm{e}^{-25}$, one-way ANOVA, ${ }^{*} P<$ 0.05; day 3-day 4: HF-PNA5 mice, $-1.5 \pm 3.5$; HF-saline mice, $11.3 \pm 4.3 ; F(1,116)=5.19, P=0.024$, one-way ANOVA, $* P<0.05)$.

Reference memory on the probe trial was assessed the day immediately after the end of the acquisition phase. Reference memory is assessed by preference for the platform area or target area when the platform is removed. Treatment with PNA5 rescued reference memory. As seen in Fig. 6C, treatment with PNA5 ( $n=11)$ significantly increased the time spent in the target quadrant compared with saline treatment $(n=10$; HF-PNA5 mice, $10.2 \pm 1.1$; HF-saline mice, $6.6 \pm 1.1$; $F(1,56)=5.6, P=0.02$, one-way ANOVA, $* P<0.05)$.

To determine whether the poor performance of HF-saline mice was possibly due to differences in visual perception or swim speed differences, both the HF and control groups were 
A

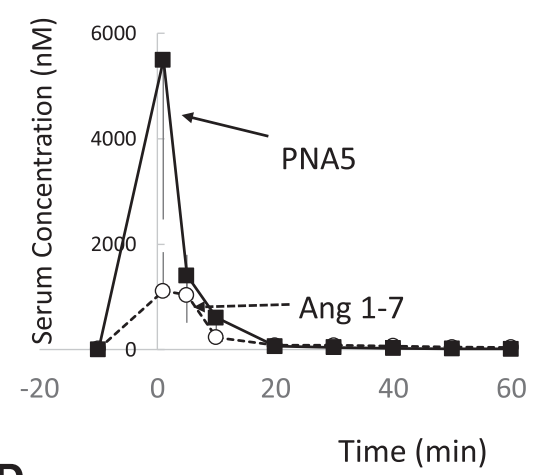

D

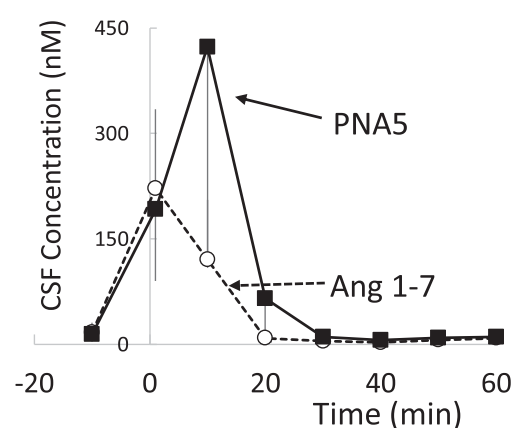

B

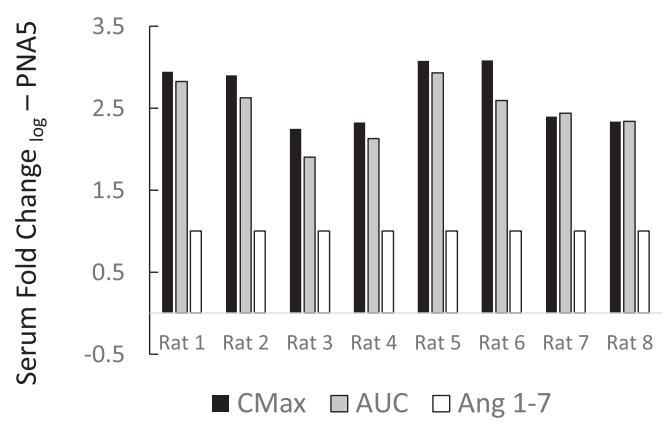

E

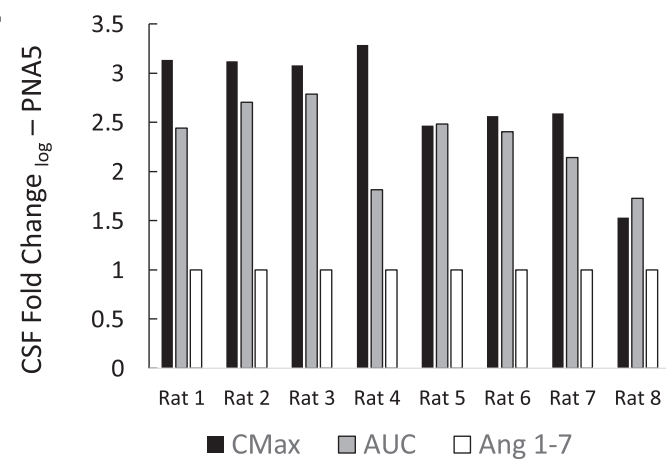

C

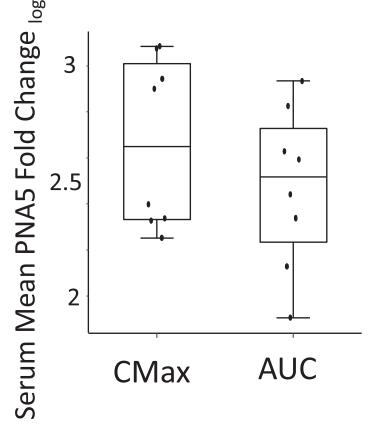

$\mathbf{F}$

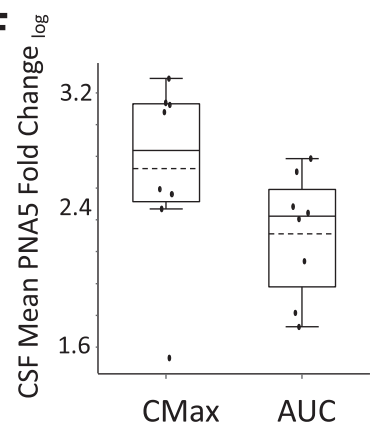

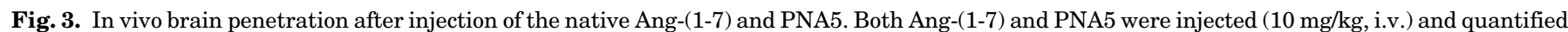

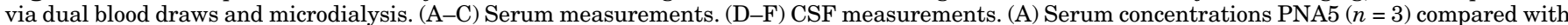

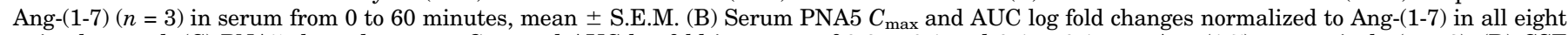

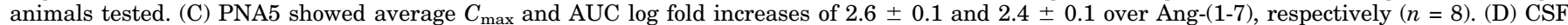

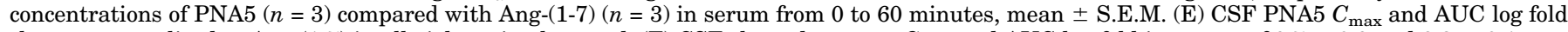

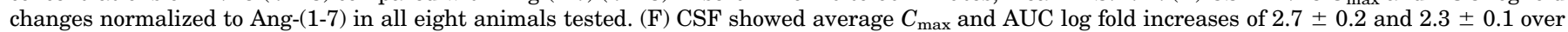
Ang-(1-7), respectively $(n=8)$.

tested for visual acuity and their average swim speeds were compared. As seen in Fig. 6D, the average swim speed of the HF-PNA5 $(n=11)$, HF-saline $(n=10)$, and control $(n=6)$ mice at 8 weeks after MI surgery were similar, suggesting that $\mathrm{HF}$ induced a reduction in performance of the MWM swim task that was not due to differences in swimming speed, but rather due to spatial memory impairment.

Likewise, as seen in Fig. 6E, both the HF and control mice were equally able to find the visible elevated platform, suggesting that the visual perception is similar in $\mathrm{HF}$ and control mice.

PNA5 Inhibition of ROS Production. Activation of the MasR has been shown by others to involve the inhibition of ROS formation, especially in vascular endothelial cells (Liu et al., 2012, 2016; Santos et al., 2013, 2017; Chen et al., 2017). To explore the cellular mechanisms underlying the ability of PNA5 to protect cognitive function, we examined the dose-dependent effects of PNA5 on ROS generation in HUVECs, and the role of the MasR activation on these effects of PNA5. As seen in Fig. 7, PNA5 MasR activation inhibits ROS formation. ROS production was measured via DHE (5 $\mu \mathrm{M}, 30$ minutes) fluorescence, which was measured using confocal microscopy (Fig. 7A). ROS production was induced by incubating HUVECs in angiotensin II (Ang II) (200 nM) for 8 hours. PNA5 $(0.1,1.0$, or $2.0 \mu \mathrm{M})$ or the MasR antagonist A779 (5-L-isoleucine-7-D-alanine-1-7-Ang II) $(2 \mu \mathrm{M})$ was added 5 hours prior to Ang II. PNA5 significantly inhibited ROS generation at all concentrations tested. As seen in Fig. 7B,
PNA5 resulted in a dose-dependent inhibition of ROS production. The MasR antagonist A779 blocked the effects of the PNA5 MasR agonist ( $* P<0.05$, one-way ANOVA, Bonferroni post hoc test), suggesting that PNA5 activation of the MasR inhibits ROS production.

Identification of Circulating Inflammatory Biomarkers. A key long-term goal of our efforts to develop PNA5 as a possible therapy for cognitive protection is to identify circulating biomarkers that correlate with inflammation-related cognitive impairment and treatment with PNA5. These data may ultimately lead to patient stratification to identify responders and nonresponders to PNA5 treatment. To that end, and to determine the early inflammatory response during the progression of VCID/HF and the early effects of 1 week of PNA5 treatment, we used the MILLIPLEX MAP Mouse High Sensitivity Multiplex Immunoassay to measure differences in circulating inflammatory profiles in animals 2 weeks post-MI and after 1 week of PNA5 treatment. Serum samples were obtained 24 hours after the last PNA5 treatment. As seen in Fig. 8, using this Multiplex Immunoassay we were able to analyze the effects of both $\mathrm{HF}$ and PNA5 treatment on a wide array of low-, medium-, and high-expressing cytokines. As seen in Fig. 9, PNA5 significantly inhibited TNF- $\alpha$ (Fig. 9A) (HF-PNA5 mice, $1.04 \pm 0.5$ $\mathrm{pg} / \mathrm{ml}$; HF-saline mice, $\left.3.9 \pm 1.2 \mathrm{pg} / \mathrm{ml} ; P=0.03,{ }^{*} P<0.05\right)$, IL-7 (Fig. 9B) (HF-PNA5 mice, $2.04 \pm 0.5 \mathrm{pg} / \mathrm{ml}$; HF-saline mice, $4.4 \pm 0.6 \mathrm{pg} / \mathrm{ml}, P=0.01, * P<0.05)$ and GCSF (Fig. 9C) (HF-PNA5 mice, $239.0 \pm 10.3 \mathrm{pg} / \mathrm{ml}$; HF-saline mice, 


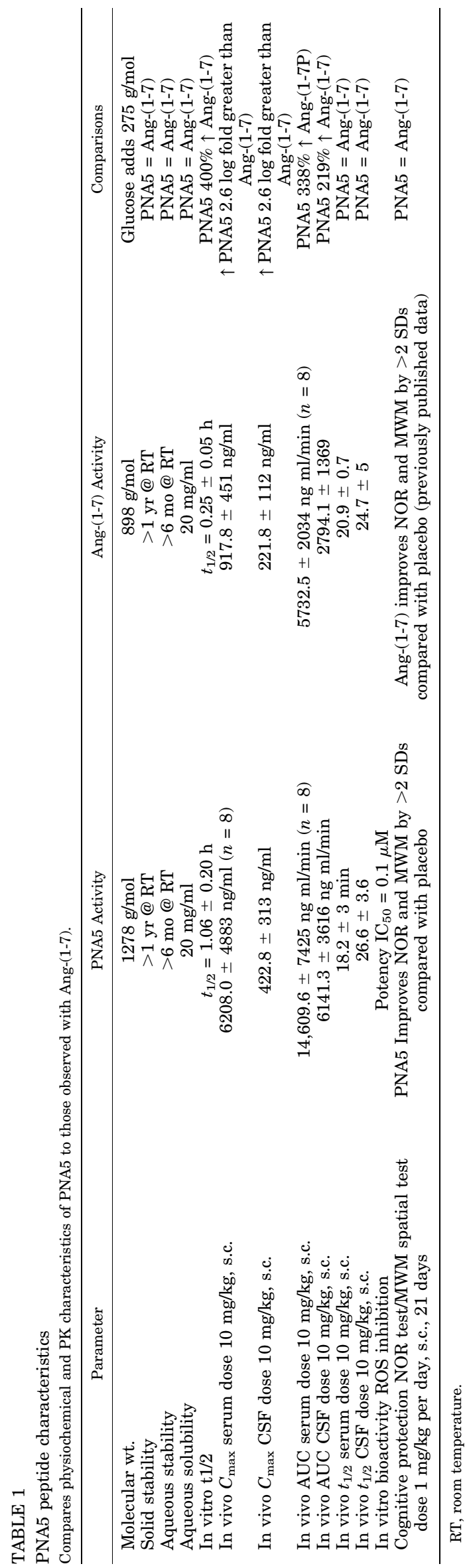

$436.2 \pm 77.4, P=0.01, * P<0.05)$ compared with salinetreated HF animals. HF alone (HF-saline) increased IL-6 (Fig. 9D) compared with controls. PNA5 trended to inhibit PNA6 but did not reach significance (HF-PNA5 mice, $9.2 \pm$ $1.8 \mathrm{pg} / \mathrm{ml}$; HF-saline mice, $15.8 \pm 6.1 \mathrm{pg} / \mathrm{ml}$; control mice, $1.2 \pm 0.6$; control vs. HF-saline mice, $P=0.02$, $* P<0.05)$, and MIP1a (Fig. 9E) (HF-PNA5 mice, $70.8 \pm 11 \mathrm{pg} / \mathrm{ml}$; HF-saline mice, $67.9 \pm 6 \mathrm{pg} / \mathrm{ml}$; control mice, $32.6 \pm 9$; control vs. HF-saline mice, $P=0.01,{ }^{*} P<0.05$ ). HF alone (HF-saline) increased MIP2 (Fig. 9F) compared with controls, and PNA5 increased MIP2 further than HF alone (HF-saline) (HF-PNA5 mice, $225.6 \pm 24 \mathrm{pg} / \mathrm{ml}$; HF-saline mice, $155.8 \pm 17 \mathrm{pg} / \mathrm{ml}$; control mice, $65.0 \pm 11$; HF-saline vs. control mice, $P=0.002$; HF-PNA5 vs. HF-saline mice, $P=0.04, * P<0.05)$.

As seen in Fig. 10, PNA5 significantly increased antiinflammatory cytokine IL-10 (Fig. 10A) (HF-PNA5 mice, $25.2 \pm 1.4 \mathrm{pg} / \mathrm{ml} ; \mathrm{HF}$-saline mice, $18.4 \pm 2.1 \mathrm{pg} / \mathrm{ml} ; P=0.02$, $* P<0.05)$ compared with saline-treated HF animals. PNA5 significantly increased the ratio of IL-10/TNF- $\alpha$ (Fig. 10B) (HF-PNA5 ratio, $17.8 \pm 5.3$; HF-saline, $4.8 \pm 0.7 ; P=0.03$, $* P<0.05)$. Differences between groups were analyzed by using a Student's $t$ test or one-way ANOVA. All statistical tests and $P$ values were calculated using Microsoft Excel with Daniel's XL Toolbox, and $\alpha$ was set at the 0.05 level. Error bars represent the S.E.M.

PNA5 and Brain Inflammation. In an effort to determine whether our model of VCID/HF-induced cognitive impairment results in increased markers of brain inflammation and whether PNA5 modifies those effects, we used immunohistochemistry to semiquantitatively measure brain Iba-1 protein expression. As seen in Fig. 11, B and $\mathrm{C}$, animals with VCID/HF show increased Iba-1 immunostaining in the cortical meninges compared with control animals (Fig. 11D). Treatment with PNA5 decreases Iba-1 immunostaining in the cortical meningeal region of the brain (Fig. 11A). Daily treatment with PNA5 for 3 weeks inhibited Iba-1 immunohistochemical expression found in cells within the meningeal surface layer (Fig. 11E) (HFPNA5 mice, $34.6 \pm 5$; HF-saline mice, $85.8 \pm 19$; control mice, $37.4 \pm 8 ; P=0.01, * P<0.05)$. It is unknown whether these Iba-1 stained cells are resident microglia or peripheral macrophages. Furthermore, these data were obtained 10 days after the final PNA5 injection, suggesting that the effects of PNA5 to inhibit brain inflammation as measured by Iba-1-expressing cells in the meninges persists long after the peptide is known to be cleared from the CSF (see Fig. 3).

\section{Discussion}

In this study we present evidence that the glucoside derivative of Ang-(1-7), the O-linked glycopeptide PNA5 1) has increased blood-brain permeability, 2) increased stability and bioavailability compared with the native Ang(1-7) peptide, and 3) inhibits ROS formation in human endothelial cells via MasR activation. Finally, we show that 3 weeks of systemic treatment with PNA5 via daily subcutaneous injection in our mouse model of VCID/HF rescues cognitive impairment and results in a sustained decrease in systemic and brain inflammation. The observed sustained reversal of cognitive dysfunction suggests that PNA5 may 


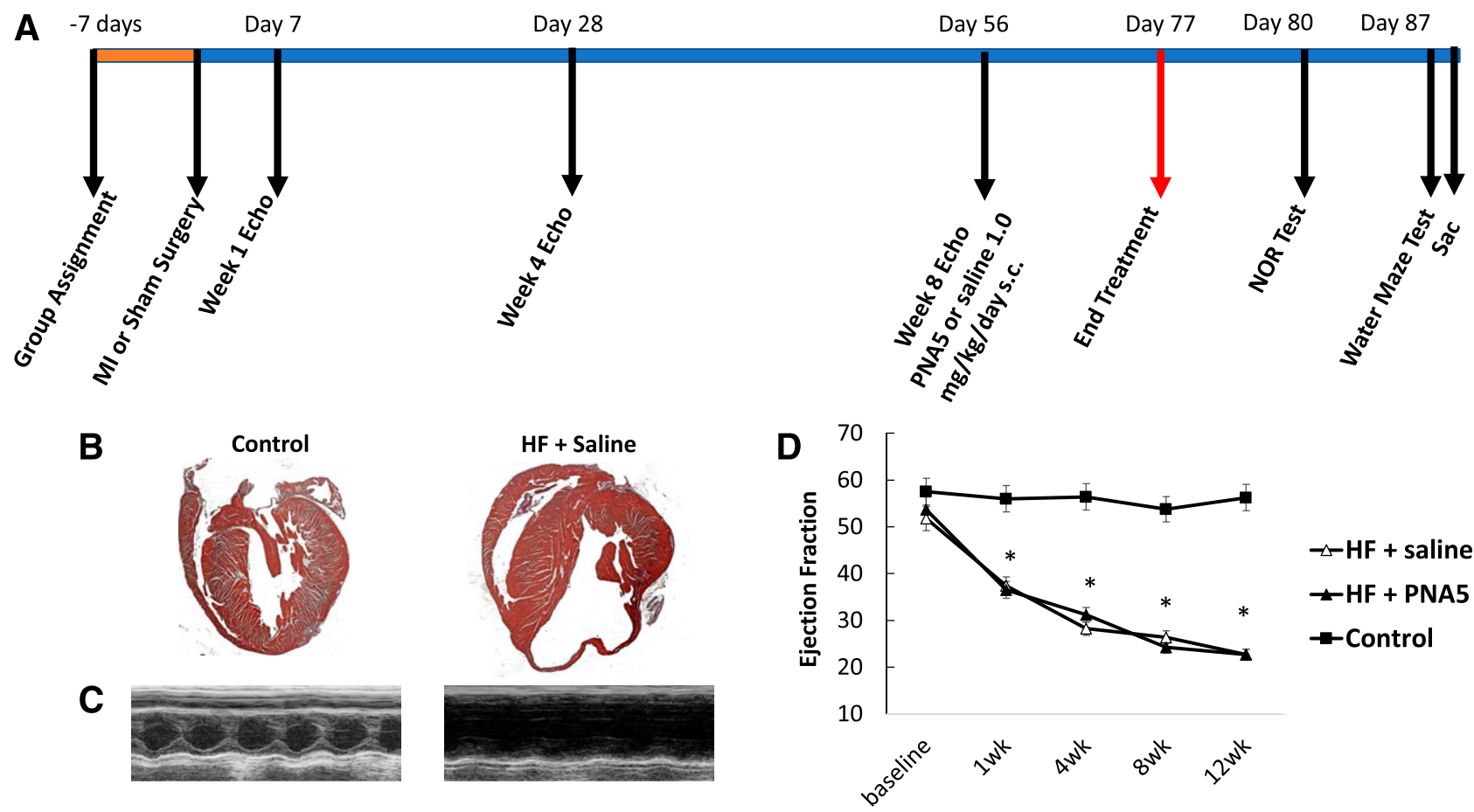

Fig. 4. Time line for the course of the experiments, and cardiac histology and morphometry in HF and control mice. (A) Representative images of H\&Estained hearts in longitudinal sections of HF and control mice. (B) Representative M-mode images of HF and control treated with either saline or PNA5. From M-mode images, parameters of ventricular chamber dimensions and function were determined. (C) EF\%. Data are presented as mean \pm S.E.M. Experimental group numbers are as follows: HF-saline, $n=10$; HF-PNA5, $n=10$; control, $n=6\left(* P<0.05\right.$ from baseline; ${ }^{* *} P<0.05$ from control group).

be a novel "first-in-class" therapy for the treatment of cognitive impairment in patients with VCID or conditions of chronic systemic inflammation and compromised brain blood flow such as that observed in patients with HF.

A New Model of VCID/HF Cognitive Impairment. Typically, VCID is used as an umbrella term that covers

A

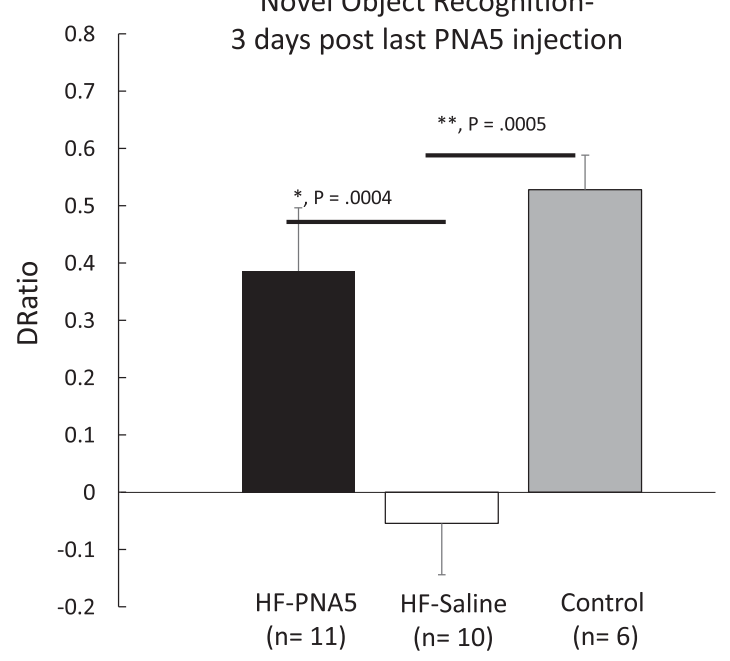

clinically observed cognitive dysfunction that is caused by cerebrovascular disease (O'Brien et al., 2003; Hachinski et al., 2006; Kalaria and Erkinjuntti, 2009; Snyder et al., 2015; Corriveau et al., 2016; Gorelick et al., 2017). Chronic decreases in cerebral perfusion, such as that observed in $\mathrm{HF}$ with decreased EF, is also a known cause of VCID and often is

B

\section{Familiarization Phase Object Preference}

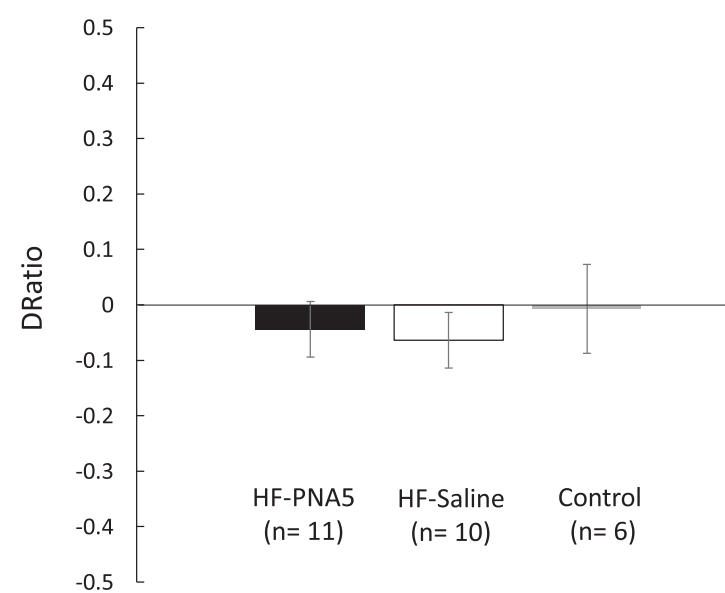

Fig. 5. Effects of PNA5 treatment on NOR task performance in HF and control mice. (A) Mean discrimination ratios, taken from the first 2 minutes of test phase, of $\mathrm{HF}$ and control mice after 3 weeks of treatment with PNA5 and 3 days after the last injection. A positive score indicates more time spent with the novel object, whereas a negative score indicates more time spent with the familiar object. A zero score indicates a null preference. PNA5 rescued object recognition performance. HF-PNA5 mice $(n=11)$ NOR discrimination ratios were similar to those of controls $(n=6)$ and significantly greater than those of the HF-saline mice $(n=10)(+0.38 \pm 0.1$ vs. $-0.05 \pm 0.09 ; F(3,28)=8.13, P=0.0004$, ANOVA, $* P<0.05)$. (B) During the familiarization phase with two identical objects, there was a null preference for the two identical objects for HF+PNA5 mice, the HF+saline mice and the control mice ( $-0.04 \pm$ $0.05,-0.06 \pm 0.1$, and $-0.007 \pm 0.1$, respectively). These results suggest that there was no difference in object preference for identical objects during the familiarization phase. 
PNA 5 Rescues Spatial Memory- 10 days post last injection

A

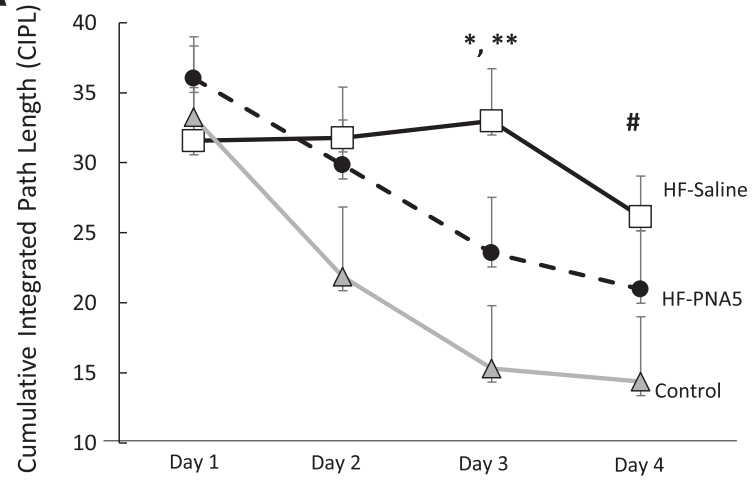

C

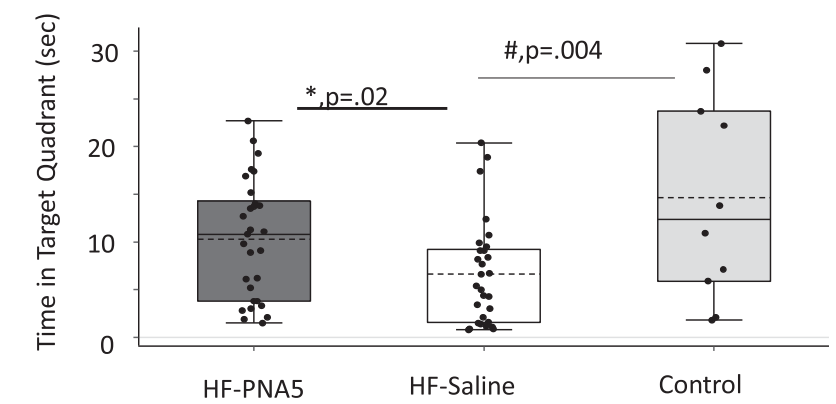

B

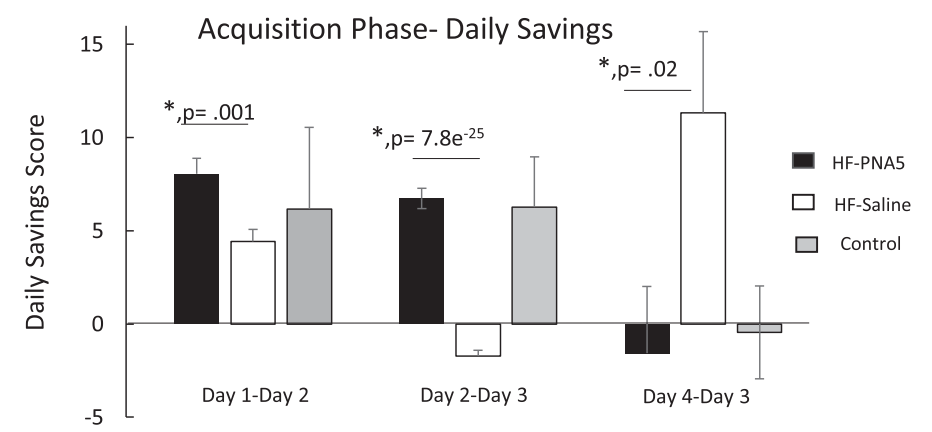

D

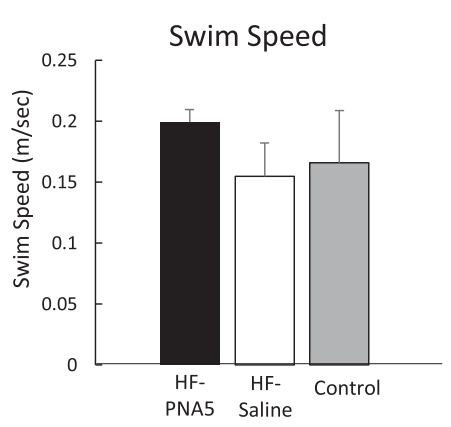

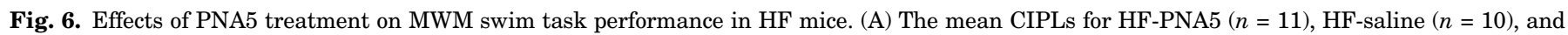

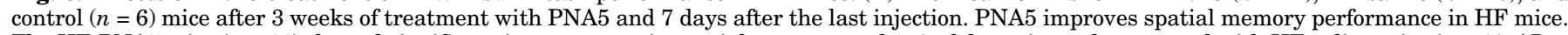

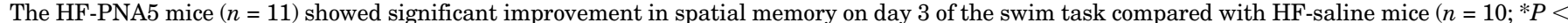

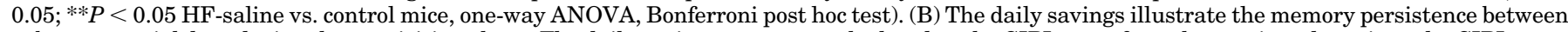

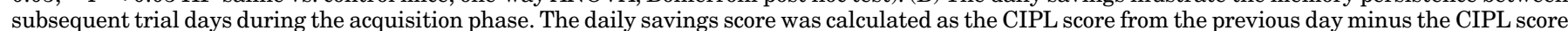

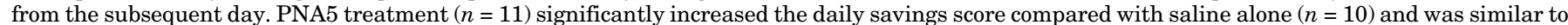

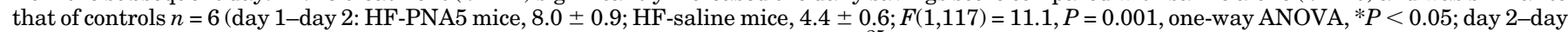

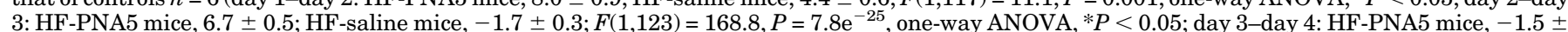

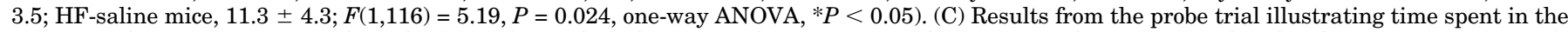

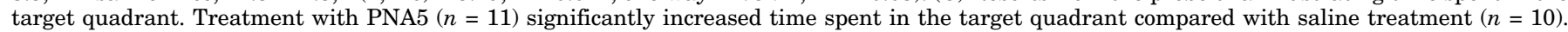

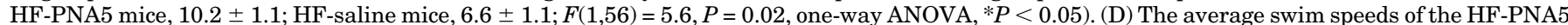

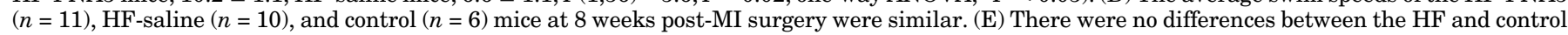
mice in the ability to find the elevated platform, suggesting that the visual acuity is the same in HF and control mice.

not accompanied by cerebral lesions or stroke (Marshall, 2012; Alosco et al., 2013; Jellinger, 2013; Justin et al., 2013; Stefansdottir et al., 2013). Indeed, there are a number of conditions that fall under the category of VCID that result in alterations to vascular perfusion of the brain and result in cognitive impairment (Capone et al., 2012). These include hypotension, cardiac arrest, cardiac arrhythmias, and chronic HF, all of which are known to result in decreased brain perfusion and cognitive impairment (Marshall, 2012; Alosco et al., 2013; Justin et al., 2013; Stefansdottir et al., 2013). In our mouse model of $\mathrm{VCID} / \mathrm{HF}, \mathrm{EF}$ decreased to approximately $37 \% 1$ week after MI and decreased further to $28 \%$ at 4 weeks post-MI and $22 \%$ at the end of 12 weeks of the study. After 12 weeks, our model results in a $30 \%$ decrease in $\mathrm{EF}$ and a significant decline in cognitive function that was accompanied increased systemic inflammation and brain Iba-1 meningeal staining consistent with brain inflammation. Our model of VCID/HF is consistent with reports in older human patients who have chronic HF with impaired $\mathrm{EF}$, who present with cognitive impairment and increased risk of $\mathrm{AD}$ (de la Torre, 2000). It has been hypothesized that there exists a Critically Attained Threshold of Cerebral
Hypoperfusion, and when this threshold is exceeded, as it is in chronic $\mathrm{HF}$ with decreased $\mathrm{EF}$, the decrease in cerebral blood results in oxygen deprivation initiating a chronic systemic and CNS inflammatory response, ultimately resulting in a decline in brain energetics and ultimately in function.

PNA5 Shows Sustained Cognitive Protective Activity. Our results suggest that PNA5, our glycosylated derivative of Ang-(1-7), not only shows improved brain penetration, selective MasR activation, and anti-inflammatory properties, but also that the cognitive protective effects of PNA5 subcutaneous daily therapy in our VCID model results in extended protection beyond the measured serum $t_{1 / 2}$ of the peptide. Indeed, we observed cognitive protection as measured in the MWM test up to 10 days past the last dose of PNA5. This extended biologic $t_{1 / 2}$ is consistent with what has been reported for other GPRC peptide agonists that activate second- and third-messenger systems, thus extending the effects of receptor activation. These results may suggest that optimal PNA5 dosing may require only once-weekly dosing after a sustained daily-loading dose. The optimization of dose frequency will be determined in future planned IND enabling studies. 
A

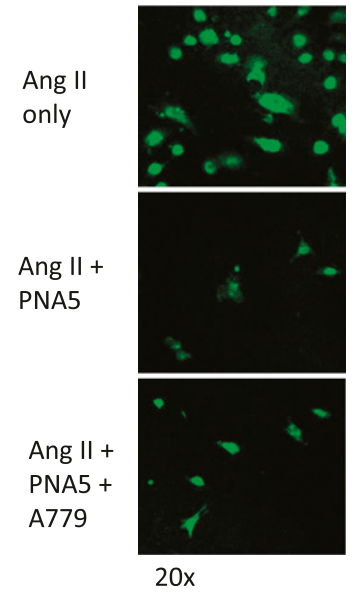

B

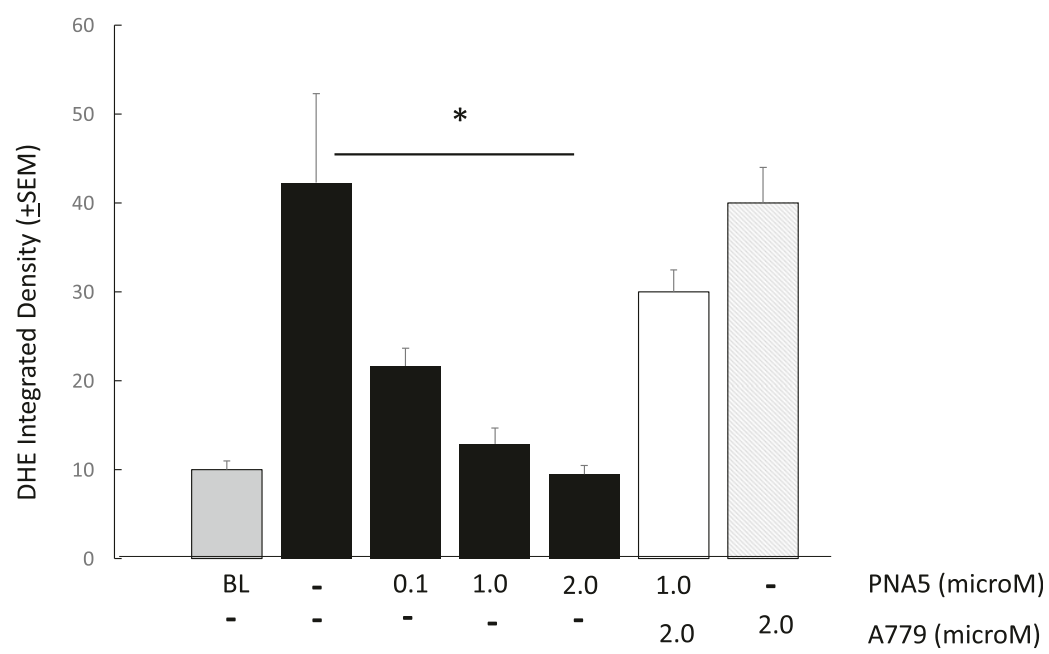

Fig. 7. PNA5 MasR activation inhibits ROS formation in HUVECs. ROS production was measured via DHE ( $5 \mu \mathrm{M}, 30$ minutes) fluorescence measured using confocal microscopy. ROS production was induced by incubating HUVECs in Ang II ( $200 \mathrm{nM})$ for 8 hours. PNA5 or the MasR antagonist ( $2 \mu \mathrm{M})$ was added 5 hours prior to Ang II. Measurements are an average of DHE integrated density from 10 to 20 cells per group. (A) Representative photomicrographs of Ang II (200 nM), Ang II + PNA5 (100 nM), and Ang II + PNA5 + A779 (2 $\mu \mathrm{M})$. (B) PNA5 significantly inhibited ROS generation at all concentrations tested. The MasR antagonist A779 blocked effects of the PNA5 MasR agonist $(* P<0.05$, one-way ANOVA, Bonferroni post hoc test).

Inflammation, VCID/HF, and Brain Function. Substantial evidence links increases in systemic inflammation to impaired cognitive function. Studies in patients with HF have shown that increases in inflammatory factors such as IL- $1 \alpha$ and IL-6 are strongly correlated with decreased cognitive performance (Ramlawi et al., 2006; Rudolph et al., 2008;
Gorelick et al., 2017). HF is also known to be associated with increases in ROS production, TNF- $\alpha$, cyclooxygenase 2 activation, and increased prostaglandin synthesis (Darley-Usmar et al., 1997; Gao et al., 2004; Felder et al., 2009). Circulating inflammatory cytokines result in a feedforward cascade that contributes to neuronal dysfunction by increasing brain ROS
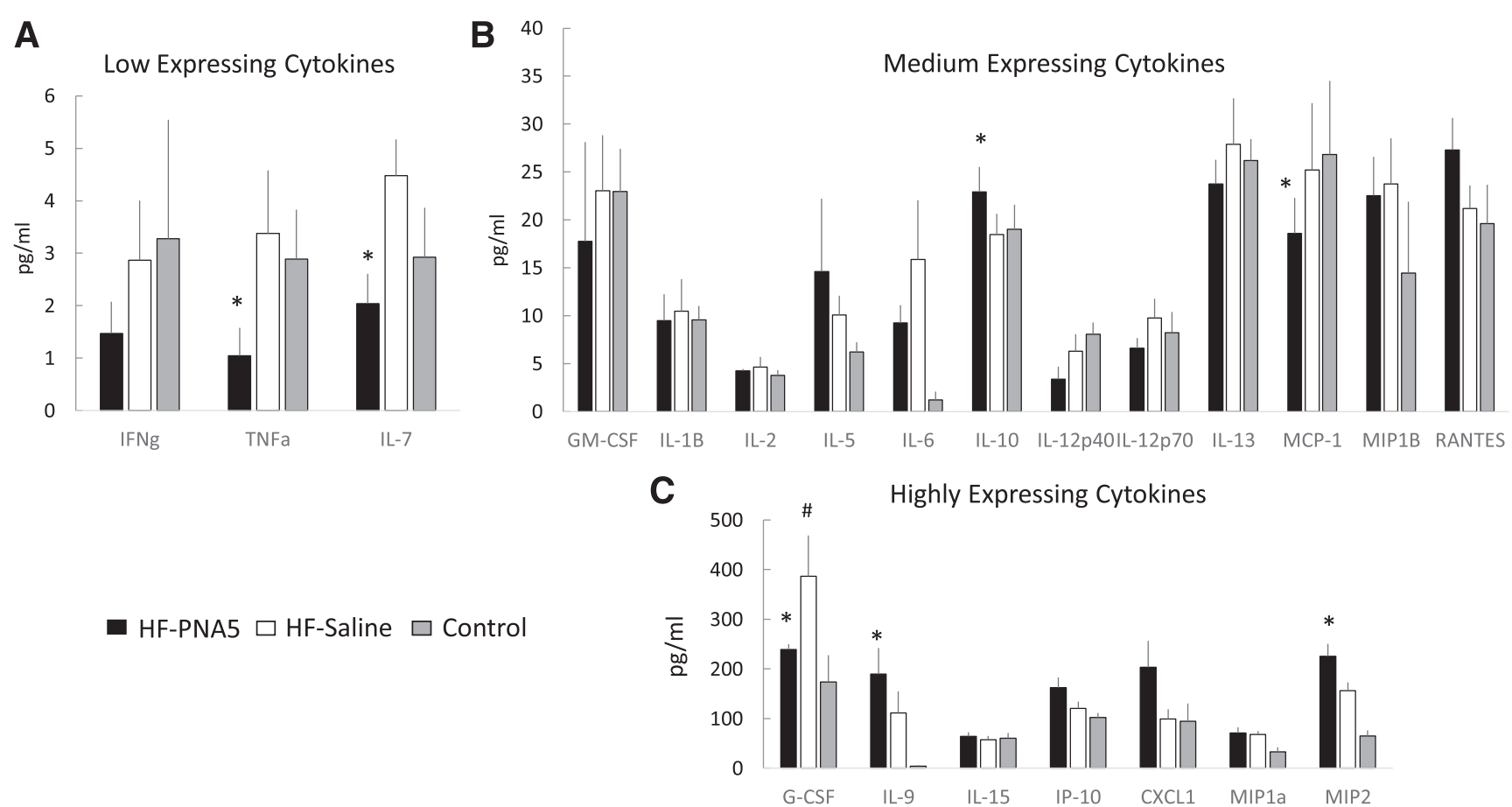

Fig. 8. Effects of PNA5 on serum inflammatory biomarkers. One week post-MI, mice were administered ( $1 \mathrm{mg} / \mathrm{kg})$ PNA5 or saline by subcutaneous injection for 1 week (three groups, $n=6-8$ each). Control mice were given saline. Two weeks post-MI, blood was collected from HF and control mice in murine EDTA tubes, centrifuged to obtain plasma, and rapidly frozen in liquid nitrogen. Plasma cytokines, chemokines, and additional inflammatory analytes were detected and quantified by multiplex immunoassay using a MAGPIX Multiplexing Instrument and accompanying Multiplex Analyst software (EMD Millipore). The results were sorted according to the level of expression of cytokines: Low (A), Medium (B), or High (C). Shown are the absolute levels of expression of each cytokine in HF-PNA5 $(n=8)$, HF-saline $(n=7)$ and control $(n=6)$ animals. $* P<0.05$ HF-PNA5 vs. PF-saline; $\# P<0.05$ HF-PNA5 vs. control; ANOVA. All samples were run in triplicate. 
A TNF-alpha

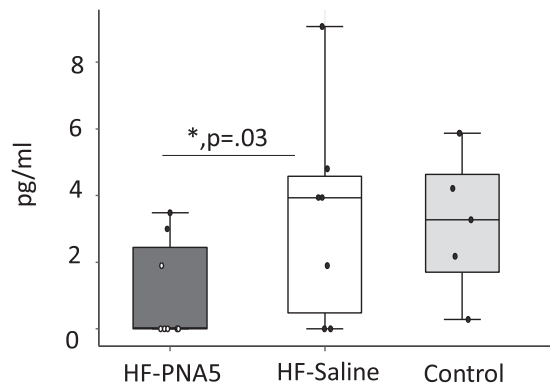

D

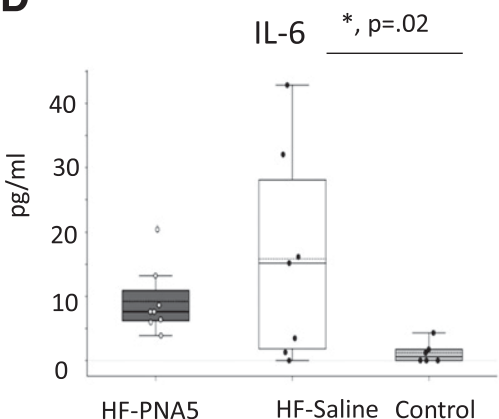

B

IL-7

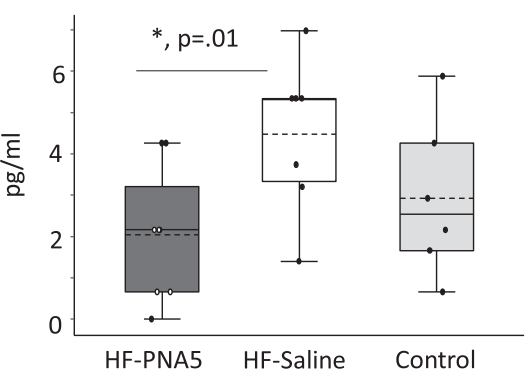

E

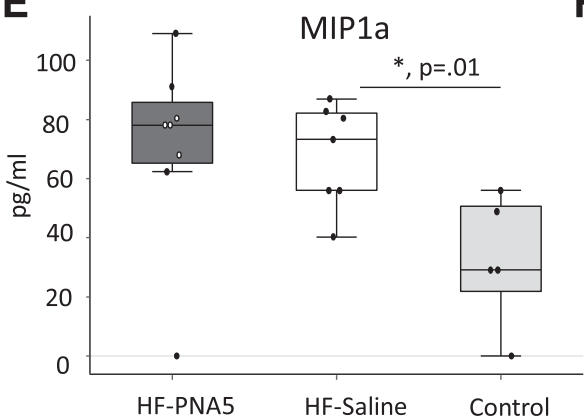

C

G-CSF

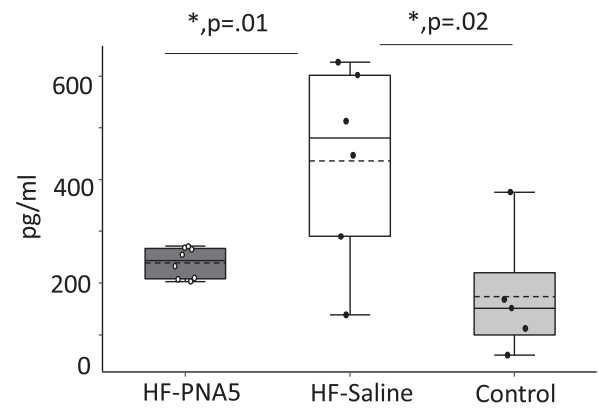

F

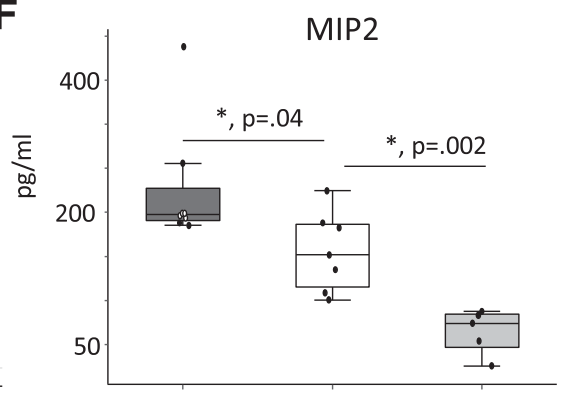

HF-PNA5 HF-Saline

Fig. 9. PNA5 inhibition of proinflammatory cytokines. Figure 9 illustrates those cytokines in Fig. 8 that showed a significant treatment effect of PNA5. PNA5 significantly inhibited TNF- $\alpha$ (HF-PNA5 mice, $1.04 \pm 0.5 \mathrm{pg} / \mathrm{ml}$; HF-saline mice, $3.9 \pm 1.2 \mathrm{pg} / \mathrm{ml} ; P=0.03 ; * P<0.05)$ (A), IL-7 (HF-PNA5 mice, $2.04+0.5 \mathrm{pg} / \mathrm{ml}$; HF-saline mice, $4.4+0.6 \mathrm{pg} / \mathrm{ml} ; P=0.01 ; * P<0.05)(B)$, and GCSF (HF-PNA5 mice, $239.0 \pm 10.3 \mathrm{pg} / \mathrm{ml} ; \mathrm{HF}-\mathrm{saline} \mathrm{mice,} 436.2 \pm 77.4 ;$ $P=0.01 ; * P<0.05)(\mathrm{C})$ compared with saline-treated HF animals. HF alone (HF-saline) increased IL-6 levels compared with those in controls (D). PNA5 trended to inhibit IL-6 but did not reach significance (HF-PNA5 mice, $9.2 \pm 1.8 \mathrm{pg} / \mathrm{ml}$; HF-saline mice, $15.8 \pm 6.1 \mathrm{pg} / \mathrm{ml}$; control mice, $1.2 \pm 0.6$; control vs. HF-saline mice, $P=0.02,{ }^{*} P<0.05$.) HF alone also increased MIP1a levels (HF-PNA5 mice, $70.8 \pm 11 \mathrm{pg} / \mathrm{ml} ; \mathrm{HF}$-saline mice, $67.9 \pm 6 \mathrm{pg} / \mathrm{ml}$; control mice, $32.6 \pm 9$; control vs. HF-saline mice, $P=0.01 ; * P<0.05$ ). HF-saline (E) increased MIP2 (F) compared with controls, and PNA5 increased MIP2 further than HF-saline (F) (HF-PNA5 mice, $225.6 \pm 24 \mathrm{pg} / \mathrm{ml}$; HF-saline mice, $155.8 \pm 17 \mathrm{pg} / \mathrm{ml}$; control mice, $65.0 \pm 11$; HF-saline vs. control mice, $P=0.002 ;$ HF-PNA5 vs. HF-saline mice, $P=0.04 ; * P<0.05$.

production and activating inflammatory pathways in the brain. This inflammatory cascade is thought to affect the entire neurovascular unit, including endothelial cells of the brain microvasculature as well as microglia, astrocytes, and neurons, and ultimately to impair cognitive function.

In the present study, we first identified circulating inflammatory biomarkers that are associated with the progression of our VCID/HF cognitive impairment model. The identification of biomarkers will be critical for the clinical development of PNA5. Importantly, clinically relevant and easy to acquire biomarkers at the onset of treatment will be necessary to identify patients likely to show positive cognitive effects. We found that after EF had been reduced to approximately $30 \%$ ( 2 weeks post-MI), there were significant increases in IL-6, IL-9, MIP1a (CCL3), MIP2 (CXC ligand 2), and GCSF in the HF-saline animals compared with controls. This change in circulating cytokine
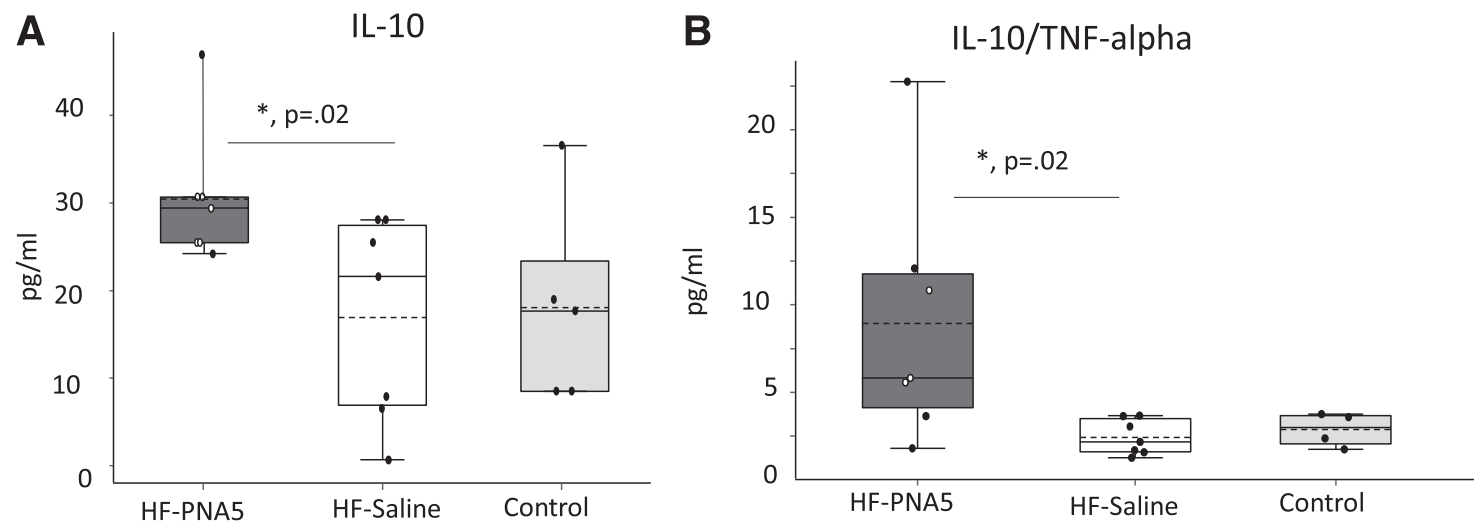

Fig. 10. PNA5 facilitation of anti-inflammatory cytokines. PNA5 significantly increased levels of the anti-inflammatory cytokine IL-10 (A) (HF-PNA5 mice, $25.2 \pm 1.4 \mathrm{pg} / \mathrm{ml}$; HF-saline mice, $18.4 \pm 2.1 \mathrm{pg} / \mathrm{ml} ; P=0.02 ; * P<0.05)$ compared with saline-treated HF animals. PNA5 significantly increased the ratio of IL-10/TNF- $\alpha$ (B) (HF/PNA5 ratio, $17.8 \pm 5.3 ; \mathrm{HF} /$ saline ratio, $4.8 \pm 0.7 ; P=0.03 ; * P<0.05)$. Differences between groups were analyzed by using a Student's $t$ test or one-way ANOVA. All statistical tests and $P$ values were calculated using Microsoft Excel with Daniel's XL Toolbox, and $\alpha$ was set at the 0.05 level. Error bars represent the S.E.M. 


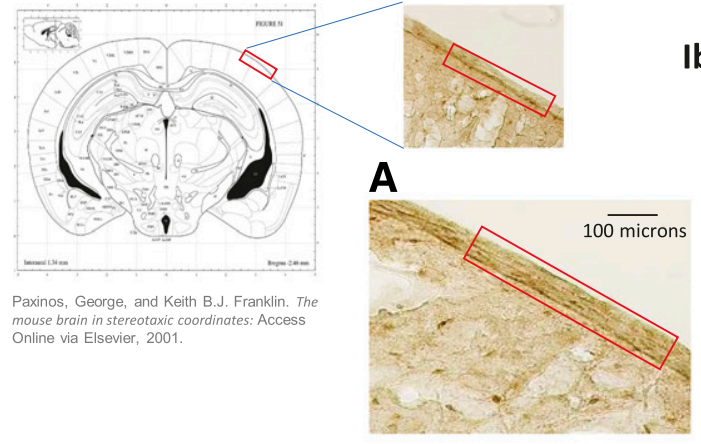

HF-PNA5

\section{Iba1-Immunohistochemistry Cortical Meningeal Surface - 7 days post last PNA5 injection}

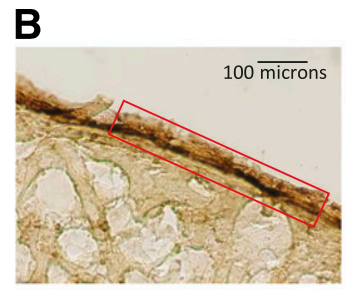

HF-Saline

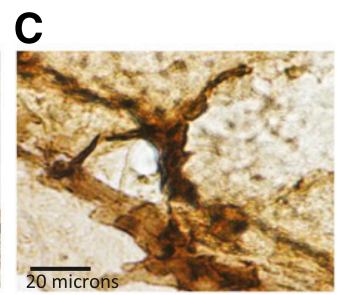

HF-Saline

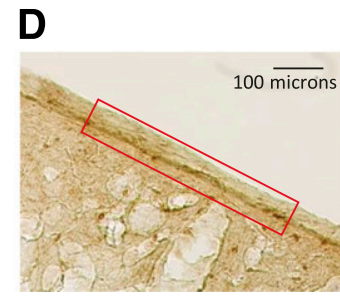

Control

$\mathbf{E}$

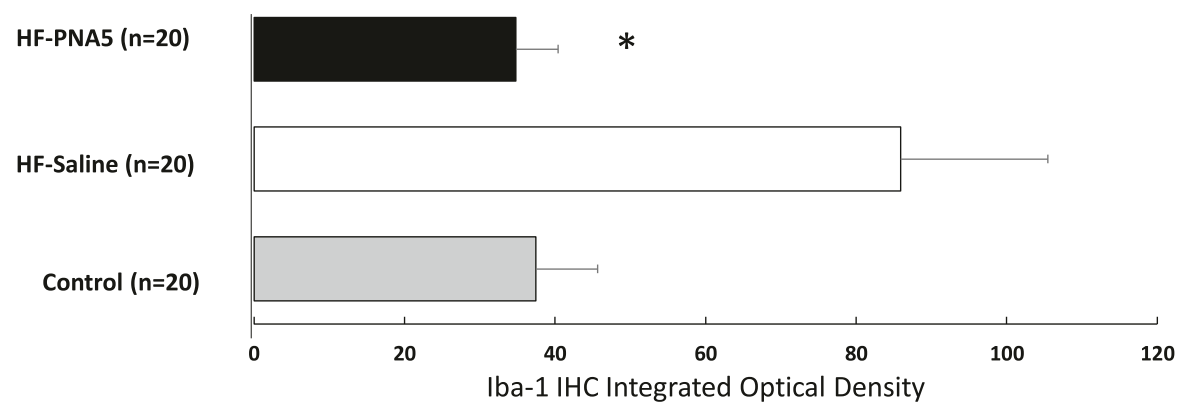

Fig. 11. Immunohistochemical analysis of the effect of PNA5 on Iba-1 levels in the brain. Quantitative analysis of Iba-1 immunohistochemical staining in the brain meningeal surface in HF-PNA5, HF-saline, and control animals 10 days after the last injection of PNA5 or saline. Daily treatment with PNA5 for 3 weeks inhibited Iba-1 immunohistochemical expression found within the meningeal surface layer. Importantly, this effect persisted 1 week after the end of PNA5 treatment, even while HF was still present. The level of DAB staining was quantified with NIH ImageJ software. The ROI manager in ImageJ was used to create a standard-sized ROI, and this same size ROI was used to analyze each slice. Identical image acquisition settings were used for all images. To ensure a uniform background across sections, we used ImageJ background subtraction prior to thresholding the images. We used an identical standard ImageJ thresholding tool setting for each image acquired. These image acquisition control techniques allowed us to successfully quantify staining that was duplicated in every image that was compared. From the threshold image, integrated density measurements from each ROI were obtained. Differences between groups were analyzed using a Student's $t$ test $(* P<0.05)$.

patterns during the development of HF has been well described by others. It has been suggested that the expression patterns of some cytokines and chemokines change during the progression of heart disease (Dick and Epelman, 2016). During the initial response to the MI, it is thought that there is an activation of cellular mechanisms needed for repair and that this initial physiologic immune response is necessary for repairing and re-establishing heart homeostasis. As the HF progresses, the physiologic inflammatory repair response converts to a pathophysiological process, leading to ventricular dysfunction and advanced cardiac disease (Mann, 2015). Thus, the inflammatory system has a dual role, including both physiologic and pathologic mechanisms (Gordon, 2008). It is this inflammatory profile and inflammatory time course in our VCID/HF mouse model that ultimately influences cognition. We have hypothesized that systemic PNA5, which has improved brain penetration compared with the native Ang-(1-7) peptide, improves cognitive function by inhibiting ROS production and this inflammatory cascade at MasR sites on both vascular endothelial cells and within the brain itself. Furthermore, Ang-(1-7) is known to improve blood flow via increases in endothelial nitric oxide release and to promote brain angiogenesis, thus improving brain oxygen delivery (Jiang et al., 2014). Both of these mechanisms would be expected to improve neuronal function and cognition.

Treatment with PNA5 inhibited the production of TNF- $\alpha$ and IL-7 and increased the IL-10/TNF- $\alpha$ ratio. TNF- $\alpha$ is a cytokine with proinflammatory properties that has a role in cognitive changes during aging. In a study of cytokine expression in the choroid plexus of patients with $\mathrm{AD}$, TNF was identified as the top upstream regulatory cytokine (Steeland et al., 2018). TNF- $\alpha$ can potentiate astroglial response to inflammation (Hensley, 2010) and induces the production of other neurodegenerative cytokines, such as IL-1, within the brain (Akiyama et al., 2000). Further, neutralization of TNF- $\alpha$ through antibodies developed for the treatment of inflammatory diseases (e.g., etanercept) reduced neurodegenerative processes (Tobinick et al., 2006; Butchart et al., 2015; Chou et al., 2016). Therefore, downregulation of TNF- $\alpha$ signaling may be a mechanism by which PNA5 improved cognitive outcomes in our HF model.

Treatment with PNA5 also increased IL-10 concentrations and the IL-10/TNF- $\alpha$ ratio. IL-10 is cytokine with antiinflammatory properties that inhibits the production of proinflammatory cytokines. Cognitive impairment in IL-10-deficient mice after exposure to an inflammatory stimuli is exacerbated (Richwine et al., 2009), and we hypothesize that PNA5 is antiinflammatory agent because of, in part, increases in IL-10 production (Liu et al., 2012, 2016; Khajah et al., 2016).

We have also shown that PNA5 inhibits Iba-1 microglia/ macrophage accumulation in the subarachnoid spaces. The protein Iba-1 is a calcium-binding protein that is known to be expressed in microglia and macrophages in the brain (Shapiro et al., 2009; Chinnery et al., 2010) in the cortical meningeal and subarachnoid regions of the brain, and shows increased 


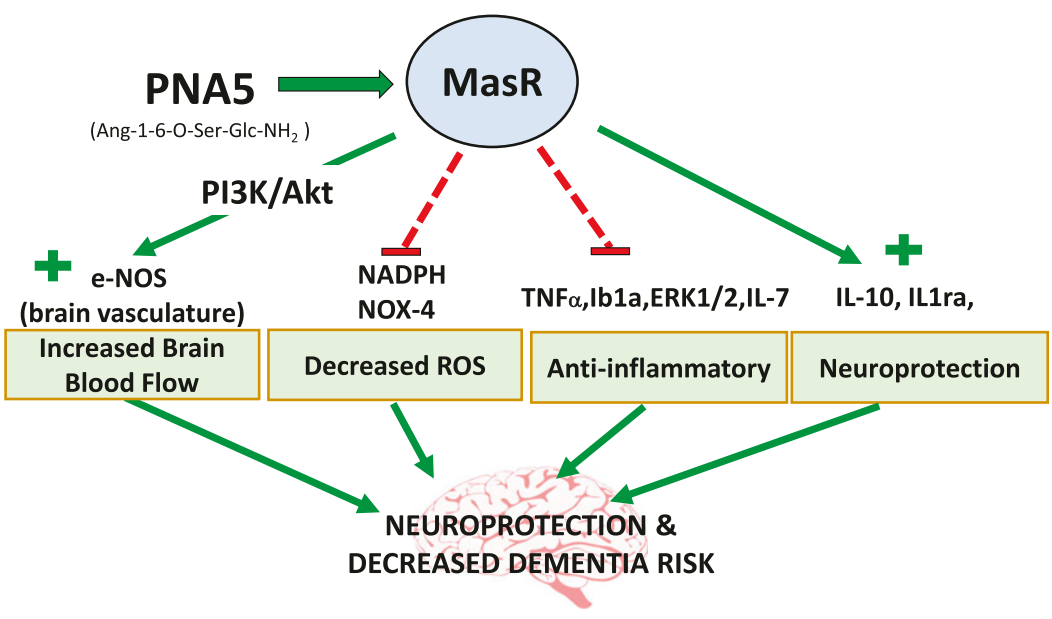

Fig. 12. Illustration of PNA5 neuroprotective pathways and of the multiple pathways by which PNA5 activation of the MasR leads to neuroprotection and decreased risk for VCID and dementia. expression during hypoxic insult, injury, and brain disease (Braun et al., 1993; Matyszak and Perry, 1996; McMenamin, 1999; Harrison-Brown et al., 2016; Prinz et al., 2017; Zrzavy et al., 2017). Microglia are known to display multiple phenotypes depending on the physiologic or pathophysiological situation. Generally, in the absence of stress or disease, microglia maintain an M2 phenotype associated with neuroprotection and repair and in the maintenance of healthy synapsis via synaptic pruning, neurogenesis, and immune surveillance. Under chronic neuroinflammatory conditions such as HF, microglia display phenotypic alterations and transition to an M1 primed state, which is proinflammatory. Microglia are activated in several neurodegenerative diseases including Parkinson's disease, $\mathrm{AD}$, and multiple sclerosis. Furthermore, microglia can be activated by hypoxia, trauma, stroke, and systemic inflammation such as that observed in patients with HF or diabetes (Durafourt et al., 2012; Melief et al., 2012). Microglia activation results in increased ROS production, cytokine production, and activation of brain inflammatory pathways that contribute to neuronal dysfunction and cognitive impairment, including memory loss (Streit et al., 2004; Hein et al., 2010; Matousek et al., 2010).

In our VCID mouse model, we observed a significant increase in Iba 1 immunoreactivity that suggests $\mathrm{HF}$ induction of M1 primed microglia in the subarachnoid regions that may be contributing to neuroinflammation and cognitive impairment. Treatment with PNA5 inhibited the Iba-1 immunoreactivity and presumably microglia activation, and this was correlated with a reversal of the cognitive impairment in our VCID model.

The studies presented here do have some limitations. First, the PK studies in CSF and plasma were performed in rats, whereas the behavior studies were performed in mice. There is some concern that the metabolism of PNA5 may differ in these two species. As we progress with the development of PNA5 for the clinic, we will fully explore the PK values and toxicology in two species as required for IND submission. Second, the use of peptides as therapeutic agents, although gaining in popularity, is not without limitations. These often include relatively short $t_{1 / 2}$ values and limited oral availability. However, as we have observed in the present study, daily treatment with PNA5 does result in sustained effects beyond the elimination of the peptide suggesting a sustained effect. In future studies, we plan on overcoming these limitations by exploring novel extended-release formulations and possible microneedle patch applications.

In conclusion, our results show that daily treatment with the glycosylated Ang-(1-7) peptide PNA5 results in the inhibition of VCID/HF-induced inflammation and rescues impaired cognitive function. These effects of PNA5 persist for at least 10 days beyond the peptide $t_{1 / 2}$. Data from this study and results by others with the native Ang-(1-7) (Ferrario, 2006; Zheng et al., 2014; Chang et al., 2016; Salminen et al., 2017) suggest that the mechanisms underlying the cognitive protective effects of PNA5 include decreases in vascular endothelial ROS, decreases in inflammatory cytokines, and improvements in cerebral blood flow. Together, these interrelated pathways work to restore health to the neurovascular unit, resulting in neuroprotection and restoration of cognitive function. Figure 12 is an illustration of the multiple pathways by which PNA5 activation of the MasR is suggested to result in neuroprotection and decreased risk for VCID and $\mathrm{AD}$ and related dementias. We suggest that PNA5 is an excellent candidate as a novel treatment of VICD and inflammatory diseases related to brain impairment.

\section{Authorship Contributions}

Participated in research design: Hay, Polt, Heien, Vanderah, Largent-Milnes, Doyle, and Konhilas.

Conducted experiments: Hay, Polt, Heien, Bartlett, Doyle, Konhilas. Performed data analysis: Hay, Polt, Heien, Bartlett, Doyle, Konhilas.

Wrote or contributed to the writing of the manuscript: Hay, Polt, Vanderah, Largent-Milnes, Rodgers, Falk, Bartlett, Doyle, and Konhilas,

\section{References}

Akiyama H, Barger S, Barnum S, Bradt B, Bauer J, Cole GM, Cooper NR, Eikelenboom P, Emmerling M, Fiebich BL, et al. (2000) Inflammation and Alzheimer's disease. Neurobiol Aging 21:383-421.

Antunes M and Biala G (2012) The novel object recognition memory: neurobiology, test procedure, and its modifications. Cogn Process 13:93-110.

Braun JS, Kaissling B, Le Hir M, and Zenker W (1993) Cellular components of the immune barrier in the spinal meninges and dorsal root ganglia of the normal rat: immunohistochemical (MHC class II) and electron-microscopic observations. Cell Tissue Res 273:209-217.

Butchart J, Brook L, Hopkins V, Teeling J, Püntener U, Culliford D, Sharples R, Sharif S, McFarlane B, Raybould R, et al. (2015) Etanercept in Alzheimer disease: a randomized, placebo-controlled, double-blind, phase 2 trial. Neurology 84: $2161-2168$

Capone C, Faraco G, Peterson JR, Coleman C, Anrather J, Milner TA, Pickel VM, Davisson RL, and Iadecola C (2012) Central cardiovascular circuits contribute to the neurovascular dysfunction in angiotensin II hypertension. J Neurosci 32: 4878-4886. 
Cermakova P, Eriksdotter M, Lund LH, Winblad B, Religa P, and Religa D (2015) Heart failure and Alzheimer's disease. J Intern Med 277:406-425.

Chang RL, Lin JW, Kuo WW, Hsieh DJ, Yeh YL, Shen CY, Day CH, Ho TJ, Viswanadha VP, and Huang CY (2016) Angiotensin-(1-7) attenuated long-term hypoxia-stimulated cardiomyocyte apoptosis by inhibiting HIF-1 $\alpha$ nuclear translocation via Mas receptor regulation. Growth Factors 34:11-18.

Chen JL, Zhang DL, Sun Y, Zhao YX, Zhao KX, Pu D, and Xiao Q (2017) Angiotensin(1-7) administration attenuates Alzheimer's disease-like neuropathology in rats with streptozotocin-induced diabetes via Mas receptor activation. Neuroscience 346:267-277.

Chinnery HR, Ruitenberg MJ, and McMenamin PG (2010) Novel characterization of monocyte-derived cell populations in the meninges and choroid plexus and their rates of replenishment in bone marrow chimeric mice. $J$ Neuropathol Exp Neurol 69:896-909.

Chou RC, Kane M, Ghimire S, Gautam S, and Gui J (2016) Treatment for rheumatoid arthritis and risk of Alzheimer's disease: a nested case-control analysis. CNS Drugs 30:1111-1120.

Corriveau RA, Bosetti F, Emr M, Gladman JT, Koenig JI, Moy CS, Pahigiannis K, Waddy SP, and Koroshetz W (2016) The science of vascular contributions to cognitive impairment and dementia (VCID): a framework for advancing research priorities in the cerebrovascular biology of cognitive decline. Cell Mol Neurobiol $\mathbf{3 6}$ 281-288.

Darley-Usmar VM, McAndrew J, Patel R, Moellering D, Lincoln TM, Jo H, Cornwell T, Digerness S, and White CR (1997) Nitric oxide, free radicals and cell signalling in cardiovascular disease. Biochem Soc Trans 25:925-929.

de la Torre JC (2000) Critically attained threshold of cerebral hypoperfusion: can it cause Alzheimer's disease? Ann N Y Acad Sci 903:424-436.

Dhanasekaran M and Polt R (2005) New prospects for glycopeptide based analgesia: glycoside-induced penetration of the blood-brain barrier. Curr Drug Deliv 2:59-73.

Dick SA and Epelman S (2016) Chronic heart failure and inflammation: what do we really know? Circ Res 119:159-176.

Durafourt BA, Moore CS, Zammit DA, Johnson TA, Zaguia F, Guiot MC, Bar-Or A and Antel JP (2012) Comparison of polarization properties of human adult microglia and blood-derived macrophages. Glia 60:717-727.

Egleton RD, Mitchell SA, Huber JD, Palian MM, Polt R, and Davis TP (2001) Improved blood-brain barrier penetration and enhanced analgesia of an opioid peptide by glycosylation. J Pharmacol Exp Ther 299:967-972.

Ennaceur A and Delacour J (1988) A new one-trial test for neurobiological studies of memory in rats. 1: behavioral data. Behav Brain Res 31:47-59.

Faul M and Coronado V (2015) Epidemiology of traumatic brain injury. Handb Clin Neurol 127:3-13.

Felder RB, Yu Y, Zhang ZH, and Wei SG (2009) Pharmacological treatment for heart failure: a view from the brain. Clin Pharmacol Ther 86:216-220.

Ferrario CM (2006) Angiotensin-converting enzyme 2 and angiotensin-(1-7): an evolving story in cardiovascular regulation. Hypertension 47:515-521.

Forte BL, Slosky LM, Zhang H, Arnold MR, Staatz WD, Hay M, Largent-Milnes TM, and Vanderah TW (2016) Angiotensin-(1-7)/Mas receptor as an antinociceptive agent in cancer-induced bone pain. Pain 157:2709-2721.

Gallagher M, Burwell R, and Burchinal M (1993) Severity of spatial learning impairment in aging: development of a learning index for performance in the Morris water maze. Behav Neurosci 107:618-626.

Gao L, Wang W, Li YL, Schultz HD, Liu D, Cornish KG, and Zucker IH (2004) Superoxide mediates sympathoexcitation in heart failure: roles of angiotensin II and $\mathrm{NAD}(\mathrm{P}) \mathrm{H}$ oxidase. Circ Res 95:937-944.

Gao XM, Dart AM, Dewar E, Jennings G, and Du XJ (2000) Serial echocardiographic assessment of left ventricular dimensions and function after myocardial infarction in mice. Cardiovasc Res 45:330-338.

Gordon S (2008) Elie Metchnikoff: father of natural immunity. Eur J Immunol $\mathbf{3 8}$ $3257-3264$

Gorelick PB, Furie KL, Iadecola C, Smith EE, Waddy SP, Lloyd-Jones DM, Bae HJ, Bauman MA Dichoans M, Duncan PW, et al. American Heart Association/ American Stroke Association (2017) Defining optimal brain health in adults: a presidential advisory from the American Heart Association/American Stroke Association. Stroke 48:e284-e303.

Harrison-Brown M, Liu GJ, and Banati R (2016) Checkpoints to the brain: directing myeloid cell migration to the central nervous system. Int J Mol Sci 17: E2030.

Hay M, Vanderah TW, Samareh-Jahani F, Constantopoulos E, Uprety AR, Barnes CA, and Konhilas J (2017) Cognitive impairment in heart failure: a protective role for angiotensin-(1-7), Behav Neurosci 131:99-114.

Heien ML (2015) Response to comment on "improved calibration of voltammetric sensors for studying pharmacological effects on dopamine transporter kinetics in vivo". ACS Chem Neurosci 6:1652-1656.

Hein AM, Stasko MR, Matousek SB, Scott-McKean JJ, Maier SF, Olschowka JA Costa AC, and O'Banion MK (2010) Sustained hippocampal IL-1beta overexpression impairs contextual and spatial memory in transgenic mice. Brain Behav Immun 24:243-253.

Hensley K (2010) Neuroinflammation in Alzheimer's disease: mechanisms, pathologic consequences, and potential for therapeutic manipulation. $J$ Alzheimers Dis 21:1-14

Jiang T, Yu JT, Zhu XC, Zhang QQ, Tan MS, Cao L, Wang HF, Lu J, Gao Q, Zhang $\mathrm{YD}$, et al. (2014) Angiotensin-(1-7) induces cerebral ischemic tolerance by promoting brain angiogenesis in a Mas/eNOS-dependent pathway. $\mathrm{Br} J$ Pharmacol 171:4222-4232.

Jones EM and Polt R (2015) CNS active O-linked glycopeptides. Front Chem 3:40.

Kass DA, Hare JM, and Georgakopoulos D (1998) Murine cardiac function: a cautionary tail. Circ Res 82:519-522.

Khajah MA, Fateel MM, Ananthalakshmi KV, and Luqmani YA (2016) Antiinflammatory action of angiotensin 1-7 in experimental colitis. PLoS One 11: e0150861.
Lefever M, Li Y, Anglin B, Muthu D, Giuvelis D, Lowery JJ, Knapp BI, Bidlack JM, Bilsky EJ, and Polt R (2015) Structural requirements for CNS active opioid glycopeptides. J Med Chem 58:5728-5741.

Liu C, Lv XH, Li HX, Cao X, Zhang F, Wang L, Yu M, and Yang JK (2012) Angiotensin-(1-7) suppresses oxidative stress and improves glucose uptake via Mas receptor in adipocytes. Acta Diabetol 49:291-299.

Liu M, Shi P, and Sumners C (2016) Direct anti-inflammatory effects of angiotensin(1-7) on microglia. J Neurochem 136:163-171.

Love S and Miners JS (2016) Cerebral hypoperfusion and the energy deficit in Alzheimer's disease. Brain Pathol 26:607-617.

Lowery JJ, Yeomans L, Keyari CM, Davis P, Porreca F, Knapp BI, Bidlack JM, Bilsky EJ, and Polt R (2007) Glycosylation improves the central effects of DAMGO. Chem Biol Drug Des 69:41-47.

Mabrouk OS, Falk T, Sherman SJ, Kennedy RT, and Polt R (2012) CNS penetration of the opioid glycopeptide MMP-2200: a microdialysis study. Neurosci Lett 531: 99-103.

Malakoutikhah M, Teixidó M, and Giralt E (2008) Toward an optimal blood-brain barrier shuttle by synthesis and evaluation of peptide libraries. J Med Chem $\mathbf{5 1}$ $4881-4889$

Mann DL (2015) Innate immunity and the failing heart: the cytokine hypothesis revisited. Circ Res 116:1254-1268.

Matousek SB, Hein AM, Shaftel SS, Olschowka JA, Kyrkanides S, and O'Banion MK (2010) Cyclooxygenase-1 mediates prostaglandin E(2) elevation and contextua memory impairment in a model of sustained hippocampal interleukin-1beta expression. $J$ Neurochem 114:247-258.

Matyszak MK and Perry VH (1996) The potential role of dendritic cells in immunemediated inflammatory diseases in the central nervous system. Neuroscience $\mathbf{7 4}$ : 599-608.

McMenamin PG (1999) Distribution and phenotype of dendritic cells and resident tissue macrophages in the dura mater, leptomeninges, and choroid plexus of the rat brain as demonstrated in wholemount preparations. J Comp Neurol 405: $553-562$

Melief J, Koning N, Schuurman KG, Van De Garde MD, Smolders J, Hoek RM, Van Eijk M, Hamann J, and Huitinga I (2012) Phenotyping primary human microglia: tight regulation of LPS responsiveness. Glia 60:1506-1517.

Miners JS, Palmer JC, and Love S (2016) Pathophysiology of hypoperfusion of the precuneus in early Alzheimer's disease. Brain Pathol 26:533-541.

Mordwinkin NM, Russell JR, Burke AS, Dizerega GS, Louie SG, and Rodgers KE (2012) Toxicological and toxicokinetic analysis of angiotensin (1-7) in two species. J Pharm Sci 101:373-380.

Moser MB, Moser EI, Forrest E, Andersen P, and Morris RG (1995) Spatial learning with a minislab in the dorsal hippocampus. Proc Natl Acad Sci USA 92:9697-9701.

Nation DA, Tan A, Dutt S, McIntosh EC, Yew B, Ho JK, Blanken AE, Jang JY, Rodgers KE, and Gaubert A (2018) Circulating progenitor cells correlate with memory, posterior cortical thickness, and hippocampal perfusion. J Alzheimers Dis 61:91-101.

Pavol MA, Willey JZ, Wei Y, Yuzefpolskaya M, Marshall RS, Marascalco PJ, Harwood J, and Lazar RM (2018) Does cognition improve following LVAD implantation? Gen Thorac Cardiovasc Surg 66:456-463.

Price BR, Norris CM, Sompol P, and Wilcock DM (2018) An emerging role of astrocytes in vascular contributions to cognitive impairment and dementia. $J$ Neurochem 144:644-650.

Prinz M, Erny D, and Hagemeyer N (2017) Ontogeny and homeostasis of CNS myeloid cells. Nat Immunol 18:385-392.

Pullicino PM, Wadley VG, McClure LA, Safford MM, Lazar RM, Klapholz M, Ahmed A, Howard VJ, and Howard G (2008) Factors contributing to global cognitive impairment in heart failure: results from a population-based cohort. J Card Fail 14: 290-295

Qiu C, Winblad B, Marengoni A, Klarin I, Fastbom J, and Fratiglioni L (2006) Heart failure and risk of dementia and Alzheimer disease: a population-based cohort study. Arch Intern Med 166:1003-1008.

Ramlawi B, Rudolph JL, Mieno S, Feng J, Boodhwani M, Khabbaz K, Levkoff SE, Marcantonio ER, Bianchi C, and Sellke FW (2006) C-Reactive protein and inflammatory response associated to neurocognitive decline following cardiac surgery. Surgery 140:221-226.

Richwine AF, Sparkman NL, Dilger RN, Buchanan JB, and Johnson RW (2009) Cognitive deficits in interleukin-10-deficient mice after peripheral injection of lipopolysaccharide. Brain Behav Immun 23:794-802.

Rodgers KE, Oliver J, and diZerega GS (2006) Phase I/II dose escalation study of angiotensin 1-7 [A(1-7)] administered before and after chemotherapy in patients with newly diagnosed breast cancer. Cancer Chemother Pharmacol 57:559-568.

Rudolph JL, Ramlawi B, Kuchel GA, McElhaney JE, Xie D, Sellke FW, Khabbaz K, Levkoff SE, and Marcantonio ER (2008) Chemokines are associated with delirium after cardiac surgery. J Gerontol A Biol Sci Med Sci 63:184-189.

Salminen A, Kauppinen A, and Kaarniranta K (2017) Hypoxia/ischemia activate processing of amyloid precursor protein: impact of vascular dysfunction in the pathogenesis of Alzheimer's disease. J Neurochem 140:536-549.

Santos CY, Snyder PJ, Wu WC, Zhang M, Echeverria A, and Alber J (2017) Pathophysiologic relationship between Alzheimer's disease, cerebrovascular disease, and cardiovascular risk: a review and synthesis. Alzheimers Dement (Amst) 7:69-87.

Santos RA, Ferreira AJ, Verano-Braga T, and Bader M (2013) Angiotensinconverting enzyme 2, angiotensin-(1-7) and Mas: new players of the reninangiotensin system. $J$ Endocrinol 216:R1-R17.

Shapiro LA, Perez ZD, Foresti ML, Arisi GM, and Ribak CE (2009) Morphological and ultrastructural features of Iba1-immunolabeled microglial cells in the hippocampal dentate gyrus. Brain Res 1266:29-36

Steeland S, Gorlé N, Vandendriessche C, Balusu S, Brkic M, Van Cauwenberghe C, Van Imschoot G, Van Wonterghem E, De Rycke R, Kremer A, et al. (2018) Counteracting the effects of TNF receptor-1 has therapeutic potential in Alzheimer's disease. EMBO Mol Med 10:e8300. 
Streit WJ, Mrak RE, and Griffin WS (2004) Microglia and neuroinflammation: a pathological perspective. J Neuroinflammation 1:14.

Suzuki H, Matsumoto Y, Ota H, Sugimura K, Takahashi J, Ito K, Miyata S, Furukawa K, Arai H, Fukumoto Y, et al. (2016) Hippocampal blood flow abnormality associated with depressive symptoms and cognitive impairment in patients with chronic heart failure. Circ $J$ 80:1773-1780.

Tobinick E, Gross H, Weinberger A, and Cohen H (2006) TNF-alpha modulation for treatment of Alzheimer's disease: a 6-month pilot study. MedGenMed 8:25.

Zheng J, Li G, Chen S, Bihl J, Buck J, Zhu Y, Xia H, Lazartigues E, Chen Y, and Olson JE (2014) Activation of the ACE2/Ang-(1-7)/Mas pathway reduce oxygen-glucose deprivation-induced tissue swelling, ROS production, and cell death in mouse brain with angiotensin II overproduction. Neuroscience 273:39-51.
Zimmerman MC, Lazartigues E, Lang JA, Sinnayah P, Ahmad IM, Spitz DR, and Davisson RL (2002) Superoxide mediates the actions of angiotensin II in the central nervous system. Circ Res 91:1038-1045.

Zrzavy T, Machado-Santos J, Christine S, Baumgartner C, Weiner HL, Butovsky O, and Lassmann H (2018) Dominant role of microglial and macrophage innate immune responses in human ischemic infarcts. Brain Pathol 28:791-805.

Address correspondence to: Meredith Hay, Department of Physiology, University of Arizona College of Medicine, 1501 N. Campbell Road, Building 201, Room 4103, Tucson, AZ 85724. E-mail: mhay@arizona.edu 